\title{
Keplerian Action, Convexity Optimization, and the 4-Body Problem
}

\section{Kuo-Chang Chen*}

Department of Mathematics, National Tsing Hua University, Hsinchu, Taiwan

Received 17 August 2020; Accepted (in revised version) 18 September 2020

Dedicated to Prof. Paul H. Rabinowitz with admiration on the occasion of his 80th birthday

\begin{abstract}
In this paper we introduce a method to construct periodic solutions for the $n$-body problem with only boundary and topological constraints. Our approach is based on some novel features of the Keplerian action functional, constraint convex optimization techniques, and variational methods. We demonstrate the strength of this method by constructing relative periodic solutions for the planar four-body problem within a special topological class, and our results hold for an open set of masses.
\end{abstract}

Key Words: $n$-body problem, variational methods, periodic solutions, convex optimization.

AMS Subject Classifications: 70F10, 37J51

\section{Introduction}

The Newtonian $n$-body body problem concerns the motion of $n$ masses $m_{1}, \cdots, m_{n} \geq 0$ moving in $\mathbb{R}^{d}, d \in\{1,2,3\}$, in accordance with Newton's law of universal gravitation:

$$
m_{k} \ddot{x}_{k}=\frac{\partial}{\partial x_{k}} U(x), \quad k=1, \cdots, n,
$$

where $x_{k} \in \mathbb{R}^{d}$ is the position of $m_{k}, x=\left(x_{1}, \cdots, x_{n}\right)$, and

$$
U(x)=\sum_{i<j} \frac{m_{i} m_{j}}{\left|x_{i}-x_{j}\right|}
$$

is the (self-)potential energy. Let

$$
K(\dot{x})=\frac{1}{2} \sum_{k=1}^{n} m_{k}\left|\dot{x}_{k}\right|^{2}
$$

*Corresponding author. Email address: kchen@math.nthu.edu.tw (K.-C. Chen) 
be the kinetic energy and $L(x, \dot{x})=U(x)+K(\dot{x})$ be the Lagrangian. Eq. (1.1) are EulerLagrange equations for the action functional

$$
\mathcal{A}_{t_{0}, t_{1}}(x)=\int_{t_{0}}^{t_{1}} L(x, \dot{x}) d t, \quad x \in H_{\mathrm{loc}}^{1}\left(\mathbb{R}, \mathbb{C}^{n}\right) .
$$

The case $\mathcal{A}_{0, T}$ will be denoted by $\mathcal{A}_{T}$. Unless specified otherwise, throughout this paper a "solution" of (1.1) is referred to a "classical solution" of (1.1).

Analytic construction for periodic solutions of (1.1) is an old school, while variational approach has become a fashion since the discovery of the hip-hop orbit with four bodies [16] and the figure- 8 orbit [15] with three bodies. Their idea of imposing symmetry constraints on solution curves was subsequently applied to many other examples, some notable successes been choreographic solutions $[3,7,13,14,21,22,25,28,29]$, multiple choreographic solutions (such as the parallelogram four-body problem) [5-7], generalized hiphops [12, Section 4.2] and [30], and many other orbits with miscellaneous types of symmetries (such as symmetries with rotating circle property) [4,17-19]. Most applications rely on manipulations of some equal masses. There are some examples without restriction on equal masses: the generalized hip-hops with the Italian symmetry [12, Section 4.2], some Hill type orbits [2], retrograde orbits for the three-body problem [9,10], and certain orbits with $n$-bodies extending Euler-Moulton relative equilibria [11]. In some of these examples, simple order-two spatial symmetry were imposed without involving permutation of masses. Apart from them, to our knowledge there seems to be no substantial progress on variational constructions for periodic solutions of (1.1) with totally distinct masses.

Numerical experiments suggest that, however, many highly symmetric orbits with identical masses persist as one perturb the masses, with the only expense being the lose of some symmetry. The persistence is in fact observed in many examples for a fairly large range of masses. Some curious experiments on perturbing masses for orbits in [5] and [7, Section 5] are major incentives of our present work. Fig. 1 is a very small list of motivating examples. With totally distinct masses, manipulations with symmetries are not helpful. Direct applications of global estimates in $[9,10]$ are also not quite useful for $n$-body problems with $n \geq 4$, as to be explained later in this paper (Section 4 ). These solutions fall in certain topological families, and it is in general a difficult task to rigorously prove the existence of a real solution within a given topological family of curves. There must be some insights and artifices missing.

The purpose of this paper is to introduce a method to construct periodic solutions for the $n$-body problem with only boundary and topological constraints (Section 3). Our approach is based on some novel features of the Keplerian action functional (Section 2), some properties of the action functional (Section 4), and some constraint convex optimization techniques (Section 5). Our approach is a substantial improvement of methods in $[9,10]$, and has no restriction on equal masses. We illustrate the strength of this method by constructing relative periodic solutions for the planar four-body problem within a special topological class (Section 6). 

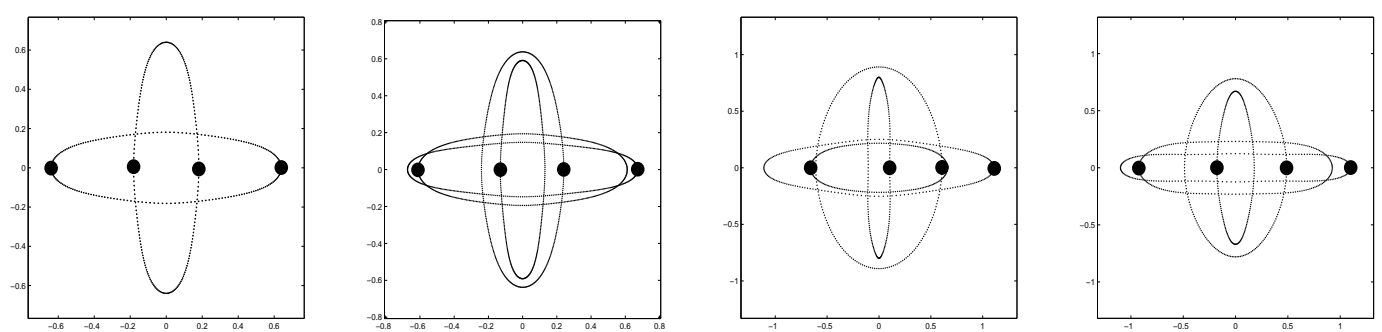

Figure 1: From left to right: The orbit in [5] with masses $\left(m_{1}, m_{2}, m_{3}, m_{4}\right)=(1,1,1,1)$, a small perturbation with masses $(0.8,0.9,1.1,1)$, and two large perturbation with masses $(1,2,3,4)$ and $(1,3,4,2)$.

\section{The Kepler problem revisited}

The Newtonian two-body problem, also called the Kepler problem, is one of the most classic problem in mechanics. Quoting Albouy [1],

“... the founding discoveries of Kepler and Newton remain strikingly beautiful, and indeed are so familiar to us that we sometimes forget that they contain surprising features."

We revisit this ancient problem to explore some simple features of the Keplerian action functional that were not found in literature, but we find fascinating and useful in our applications.

Consider the problem with masses $m_{1}, m_{2}>0$ and positions $x_{1}, x_{2} \in \mathbb{R}^{2}$. Let $\mathbf{r}=$ $x_{2}-x_{1}$ be the relative position, $\lambda>0$ be the total mass multiplied by the gravitational constant, then the equation of motion is

$$
\ddot{\mathbf{r}}=-\frac{\lambda \mathbf{r}}{|\mathbf{r}|^{3}} \text {. }
$$

A solution curve $\mathbf{r}(t)$ for the Kepler problem either traces out a conic with the origin as one of its foci, or composed of rectilinear motions with zero angular momentum. We shall identify the space containing the conic by $\mathbb{R}^{2}$ or $\mathbb{C}$ and write $\mathbf{r}$ in polar form

$$
\mathbf{r}=r e^{i \theta}, \quad r(\theta)=\frac{\mathfrak{p}}{1+\mathfrak{e} \cos \left(\theta-\theta_{0}\right)}, \quad \text { where } \mathfrak{e} \geq 0, \mathfrak{p}>0, \theta_{0} \in[0,2 \pi)
$$

For elliptical orbits (i.e., $\mathfrak{e} \in[0,1)$ ) and hyperbolic orbits (i.e., $\mathfrak{e} \in(1, \infty)$ ), the semi-latus rectum $\mathfrak{p}$, eccentricity $\mathfrak{e}$, and semi-major axis $\mathfrak{a}$ are related by $\mathfrak{p}=\mathfrak{a}\left(1-\mathfrak{e}^{2}\right)$. The constant $\theta_{0}$ is the phase angle between the major axis and the real line. by

Eq. (2.1) is the Euler-Lagrange equation of the Keplerian action functional $I_{\lambda, T}$ defined

$$
I_{\lambda, T}(\mathbf{r})=\int_{0}^{T} \frac{1}{2}|\dot{\mathbf{r}}|^{2}+\frac{\lambda}{|\mathbf{r}|} d t=\int_{0}^{T} \frac{1}{2}\left(\dot{r}^{2}+r^{2} \dot{\theta}^{2}\right)+\frac{\lambda}{r} d t, \quad \mathbf{r} \in H^{1}([0, T], \mathbb{C}) .
$$


This functional is well-known to be weakly lower semi-continuous [20], and is coercive (i.e., $I_{\lambda, T}(\mathbf{r}) \rightarrow \infty$ as $\|\mathbf{r}\|_{H^{1}} \rightarrow \infty$ ) on various subspaces.

It is often preferred to normalize relevant coefficients by suitable change of variables. For example, setting $\tilde{\mathbf{r}}(t)=\left(\mu T^{2}\right)^{-1 / 3} \mathbf{r}(t T)$, then $\mathbf{r}$ solves (2.1) with $\lambda=\mu$ if and only if $\tilde{\mathbf{r}}$ solves (2.1) with $\lambda=1$. Their action values are related by

$$
I_{\mu, T}(\mathbf{r})=\left(\mu^{2} T\right)^{\frac{1}{3}} I_{1,1}(\tilde{\mathbf{r}}) .
$$

From the perspective of the classical theory of variational calculus, one of the most natural and typical questions is to answer the minimization problem for the Keplerian action functional confined to the space

$$
\Gamma_{T}\left(\Omega_{0}, \Omega_{1}\right)=\left\{\mathbf{r} \in H^{1}([0, T], \mathbb{C}): \mathbf{r}(0) \in \Omega_{0}, \mathbf{r}(T) \in \Omega_{1}\right\},
$$

where boundary constraints $\Omega_{0}, \Omega_{1}$ are closed subsets of $\mathbb{C}$. For various boundary constraints, coercivity of $I_{\lambda, T}$ often follows easily from simple variants of Poincaré's inequality. Typical examples are: one or both of $\Omega_{0}$ and $\Omega_{1}$ are bounded, $\Omega_{0}$ and $\Omega_{1}$ are transversal lines, and so forth. Minimizers in such spaces are bound to exist. The question of our interest is therefore not about existence of minimizers, but about qualitative features of action minimizers and minimal action values. In particular, we would like to know whether or not minimizers fall inside the singular subspace; i.e., the subset of curves with collisions.

The major purpose of this section is to analyze the minimization problem

$$
\inf _{\Gamma_{T}\left(\Omega_{0}, \Omega_{1}\right)} I_{\lambda, T}
$$

for cases where $\Omega_{0}$ and $\Omega_{1}$ are either singletons or rays emanating from the origin. The most critical property for our purpose is the Theorem 2.1 in Subsection 2.3 concerning convexity and monotonicity of infimum action values.

\subsection{Minimization with two variable endpoints}

The ray and line generated by $\xi \neq 0$ are denoted respectively by

$$
\mathbb{R}_{+} \xi=\{r \xi: r \in[0, \infty)\}, \quad \mathbb{R} \xi=\{r \xi: r \in \mathbb{R}\} .
$$

For the special case $\xi \in \mathbb{R}, \xi>0$, the ray it generates is simply the positive real axis $\mathbb{R}_{+}$.

Given $\xi_{0}, \xi_{1} \neq 0$. The Keplerian action functional $I_{\lambda, T}$ attains its infimum on $\Gamma_{T}\left(\mathbb{R}_{+} \xi_{0}, \mathbb{R}_{+} \xi_{1}\right)$ if and only if the angle $\phi=\angle \xi_{0} \xi_{1}$ between $\mathbb{R}_{+} \xi_{0}$ and $\mathbb{R}_{+} \xi_{1}$ is in $(0, \pi]$ (see [8, Proposition 1] for a more general statement and its proof). Union of such spaces and their singular subspaces can be characterized by

$$
\begin{aligned}
& \Gamma_{T, \phi}:=\left\{\mathbf{r} \in H^{1}([0, T], \mathbb{C}):\langle\mathbf{r}(0), \mathbf{r}(T)\rangle=|\mathbf{r}(0)||\mathbf{r}(T)| \cos \phi\right\}, \\
& \Gamma_{T, \phi}^{*}:=\left\{\mathbf{r} \in \Gamma_{T, \phi}: \mathbf{r}(t)=0 \text { for some } t \in[0, T]\right\} .
\end{aligned}
$$


The symbol $\langle\cdot, \cdot\rangle$ stands for the standard scalar product in $\mathbb{R}^{2} \cong \mathbb{C}$. Clearly, minimization over $\Gamma_{T, \phi}$ is the same as minimization over $\Gamma_{T}\left(\mathbb{R}_{+}, \mathbb{R}_{+} e^{i \phi}\right)$.

The following proposition summarizes some discussions in [9, Section 5.1].

Proposition 2.1. Let $\phi \in(0, \pi], T>0, \lambda>0$ be constants. Let

$$
r_{\lambda, T, \phi}^{\sharp}=\left(\frac{\lambda T^{2}}{\phi^{2}}\right)^{\frac{1}{3}} .
$$

Then

$$
\begin{aligned}
& \inf _{\Gamma_{T, \phi}} I_{\lambda, T}=\frac{3}{2}(\lambda \phi)^{\frac{2}{3}} T^{\frac{1}{3}}=\frac{3 \lambda T}{2 r_{\lambda, T, \phi}^{\sharp}}, \\
& \inf _{\Gamma_{T, \phi}^{*}} I_{\lambda, T}=\frac{3}{2}(\lambda \pi)^{\frac{2}{3}} T^{\frac{1}{3}}=\frac{3 \lambda T}{2 r_{\lambda, T, \pi}^{\sharp}} .
\end{aligned}
$$

On $\Gamma_{T, \phi}$, minimizers are congruent to

$$
\mathbf{r}_{\lambda, T, \phi}^{\sharp}=r_{\lambda, T, \phi}^{\sharp} e^{\frac{t}{T} \phi i} .
$$

On $\Gamma_{T, \phi^{\prime}}^{*}$ minimizer is either collision or ejection orbit with zero velocity at one end and zero position at the other end.

\subsection{Minimization with two fixed endpoints}

The problem of transferring from one point to another in a given time is the objective of the Lambert theorem [23]. Here we are given initial position $\xi_{0}$, final position $\xi_{1}$, and transfer time $T$. The Lambert theorem provides functional equations, which express $T$ in terms of $\left|\xi_{0}\right|+\left|\xi_{1}\right|,\left|\xi_{0}-\xi_{1}\right|$, and the energy $\mathfrak{h}$. With all possible values of $\mathfrak{h}$ thus determined, the action minimizer can be selected by direct evaluation of $\mathfrak{h} T+2 \int_{0}^{T} \frac{1}{r} d t$. Explicit formulae without the integrand can be obtained, for instance, by using eccentric anomaly for elliptical orbits, or hyperbolic eccentric anomaly for hyperbolic orbits. The proof of the Lambert theorem by Lagrange [24] is much shorter and more direct. Readers are referred to [1] for a conceptual proof and more complete bibliography.

To be consistent with Eqs. (2.1) and (2.2), kinetic energy for the Kepler problem (2.1) is referred to the term $\frac{1}{2}|\dot{\mathbf{r}}|^{2}$ and the total energy $\mathfrak{h}$ is referred to

$$
\mathfrak{h}=\frac{1}{2}|\dot{\mathbf{r}}|^{2}-\frac{\lambda}{|\mathbf{r}|} .
$$

This term multiplied by the reduced mass $m_{1} m_{2} /\left(m_{1}+m_{2}\right)$ is the more standard definition of total energy.

One remarkable thing Lambert's theorem tells us is that the relation between the transfer time $T$ and energy $\mathfrak{h}$ can be completely characterized by rectilinear motions-for 
a Keplerian arc with nonzero angular moment which connects $\xi_{0}$ and $\xi_{1}$, transfer time $T$ and energy $\mathfrak{h}$ are related in exactly the same way if we replace $\left(\xi_{0}, \xi_{1}\right)$ by $\left(\xi_{0}^{\prime}, \xi_{1}^{\prime}\right) \in \mathbb{R}_{+}^{2}$ as long as $\left|\xi_{0}\right|+\left|\xi_{1}\right|=\left|\xi_{0}^{\prime}\right|+\left|\xi_{1}^{\prime}\right|$ and $\left|\xi_{0}-\xi_{1}\right|=\left|\xi_{0}^{\prime}-\xi_{1}^{\prime}\right|$. This can be extended to several other parameters. Following Albouy [1], we call real analytic functions Lambert parameters if they are defined on an open set in the space of arcs and if they satisfy three axioms:

(i) A Lambert parameter is not constant on a family of arcs with the same configuration.

(ii) If $f$ and $g$ are two Lambert parameters, then $\left|\xi_{0}\right|+\left|\xi_{1}\right|,\left|\xi_{0}-\xi_{1}\right|, f$, and $g$ are functionally dependent.

(iii) The energy $\mathfrak{h}$ is a Lambert parameter.

There are several Lambert parameters identified in [1, Proposition 31]. Apart from the transfer time, as identified by the classical Lambert theorem, what we need is just one:

Proposition 2.2 (Albouy [1]). The action integral is a Lambert parameter.

In light of this, let us focus on rectilinear paths which begin and/or end with collision. For convenience we focus on paths ejecting from the origin at $t=0$ and moving along the positive real axis $\mathbb{R}_{+}$. Let $T_{\lambda}\left(x_{0}, x_{1}, \mathfrak{h}\right)$ be the transfer time from $x_{0}$ to $x_{1}, 0 \leq x_{0} \leq x_{1}$, without passing through collision, and with prescribed energy $\mathfrak{h}$. Let $v_{1}$ be the velocity of the path at time $T_{\lambda}\left(x_{0}, x_{1}, \mathfrak{h}\right)$. Then by direct integration we have

$$
T_{\lambda}\left(x_{0}, x_{1}, \mathfrak{h}\right)=T_{\lambda}\left(0, x_{1}, \mathfrak{h}\right)-T_{\lambda}\left(0, x_{0}, \mathfrak{h}\right),
$$

where

$$
T_{\lambda}(0, x, \mathfrak{h})= \begin{cases}\frac{-\lambda}{\sqrt{-2 \mathfrak{h} h}}\left\{\sin ^{-1}\left(\sqrt{\frac{-\mathfrak{h} x}{\lambda}}\right)-\sqrt{\left.\frac{-\mathfrak{h} x}{\lambda}\left(1+\frac{\mathfrak{h} x}{\lambda}\right)\right\},}\right. & \text { if } \mathfrak{h}<0, v_{1} \geq 0, \\ \frac{-\lambda}{\sqrt{-2 \mathfrak{h} h}}\left\{\pi-\sin ^{-1}\left(\sqrt{\frac{-\mathfrak{h} x}{\lambda}}\right)+\sqrt{\left.\frac{-\mathfrak{h} x}{\lambda}\left(1+\frac{\mathfrak{h} x}{\lambda}\right)\right\},}\right. & \text { if } \mathfrak{h}<0, v_{1}<0, \\ \frac{\lambda}{\sqrt{2 \mathfrak{h} h}}\left\{-\sinh ^{-1}\left(\sqrt{\frac{\mathfrak{h} x}{\lambda}}\right)+\sqrt{\left.\frac{\mathfrak{h} x}{\lambda}\left(1+\frac{\mathfrak{h} x}{\lambda}\right)\right\},}\right. & \text { if } \mathfrak{h}>0, \\ \frac{\sqrt{2}}{3 \sqrt{\lambda}} x^{\frac{3}{2}}, & \text { if } \mathfrak{h}=0 .\end{cases}
$$

When $\mathfrak{h}<0$, the second formula of $T_{\lambda}(0, x, \mathfrak{h})$ in $(2.7 \mathrm{~b})$ represents the transfer time with non-monotonic rectilinear motion. In this case, in the first equation (2.7a) showing formula for $T_{\lambda}\left(x_{0}, x_{1}, \mathfrak{h}\right)$, we shall use the first formula in $(2.7 \mathrm{~b})$ for $T_{\lambda}\left(0, x_{0}, \mathfrak{h}\right)$. See the first graph in Fig. 2 for a typical case-the graph of $T_{1}(0,2, \mathfrak{h})$ as a function of $\mathfrak{h}$.

The energy $\mathfrak{h}$ of the Keplerian action minimizer on $\Gamma_{T}(0, \xi)$ with a prescribed transfer time $T$ is implicitly given by formulae above. The minimum action value can be calculated accordingly via direct integration. For a monotonic rectilinear motion $\mathbf{r}$ ejecting 
from the origin at $t=0$, ending with position $x>0$, its action value is given by

$$
I_{\lambda, T}(\mathbf{r})= \begin{cases}\frac{\lambda}{\sqrt{-2 \mathfrak{h}}}\left\{\sqrt{\frac{-\mathfrak{h} x}{\lambda}\left(1+\frac{\mathfrak{h} x}{\lambda}\right)}+3 \sin ^{-1}\left(\sqrt{\frac{-\mathfrak{h} x}{\lambda}}\right)\right\}, & \text { if } \mathfrak{h}<0, \\ \frac{\lambda}{\sqrt{2 \mathfrak{h}}}\left\{\sqrt{\frac{\mathfrak{h} x}{\lambda}\left(1+\frac{\mathfrak{h} x}{\lambda}\right)}+3 \sinh ^{-1}\left(\sqrt{\frac{\mathfrak{h} x}{\lambda}}\right)\right\}, & \text { if } \mathfrak{h}>0, \\ 2 \sqrt{2 \lambda x}, & \text { if } \mathfrak{h}=0 .\end{cases}
$$

Other cases can be easily derived from these formulae.

When a boundary constraint is a singleton, say $\Omega_{0}=\{\xi\}$, we shall denote the space $\Gamma_{T}\left(\{\xi\}, \Omega_{1}\right)$ by $\Gamma_{T}\left(\xi, \Omega_{1}\right)$ for simplicity, and likewise for other cases. Some special cases were summarized in the following proposition.

Proposition 2.3. Let $T>0, \lambda>0$ be constants, and let

$$
\omega_{0}=2\left(\frac{\lambda}{\pi^{2}}\right)^{\frac{1}{3}}, \omega=\left(\frac{9 \lambda}{2}\right)^{\frac{1}{3}} .
$$

Then

$$
\begin{aligned}
& \inf _{\Gamma_{T}(0,0)} I_{\lambda, T}=\frac{3}{2^{1 / 3}}(\lambda \pi)^{\frac{2}{3}} T^{\frac{1}{3}}, \\
& \inf _{\Gamma_{T}\left(0, \omega_{0} T^{2 / 3}\right)} I_{\lambda, T}=\frac{3}{2}(\lambda \pi)^{\frac{2}{3}} T^{\frac{1}{3}}=\inf _{\Gamma_{T}\left(0, \mathbb{R}_{+}\right)} I_{\lambda, T}, \\
& \inf _{\Gamma_{T}\left(0, \omega T^{2 / 3}\right)} I_{\lambda, T}=2\left(\frac{6}{\pi^{2}}\right)^{\frac{1}{3}}(\lambda \pi)^{\frac{2}{3}} T^{\frac{1}{3}} .
\end{aligned}
$$

Proof. The second identity is achieved at the ejection orbit with zero velocity at the ending point, a case included in Proposition 2.1. It also minimizes $I_{\lambda, T}$ on $\Gamma_{T}\left(0, \mathbb{R}_{+}\right)$since it is the unique monotonic ejection orbit along $\mathbb{R}_{+}$with zero velocity when $t=T$. The first identity is a simple corollary of the second. The third identity is the action value of the unique parabolic ejection orbit along the real line given by $x(t)=\omega t^{2 / 3}$. Details are simple calculations left to the reader.

For general ending point $x>0$ and prescribed transfer time $T$, the energy $\mathfrak{h}$ can approximated by finding roots of $T_{\lambda}(0, x, \mathfrak{h})-T$, where $T_{\lambda}(0, x, \mathfrak{h})$ is given by $(2.7 \mathrm{~b})$. Finding roots with any desired precision can be easily carried out by Newton's method, so the action value can be also calculated with any desired precision. The second graph in Fig. 2 is the graph of $\frac{1}{2} \inf _{\Gamma_{2}(0,2 x)} I_{1,2}$ as a function of half final position $x \in \mathbb{R}_{+}$. That is, we consider ejection orbit with final position $2 x$ at time 2 , and consider half of its action value. This function is selected because it is exactly what we shall use in the proof of Theorem 6.1. 

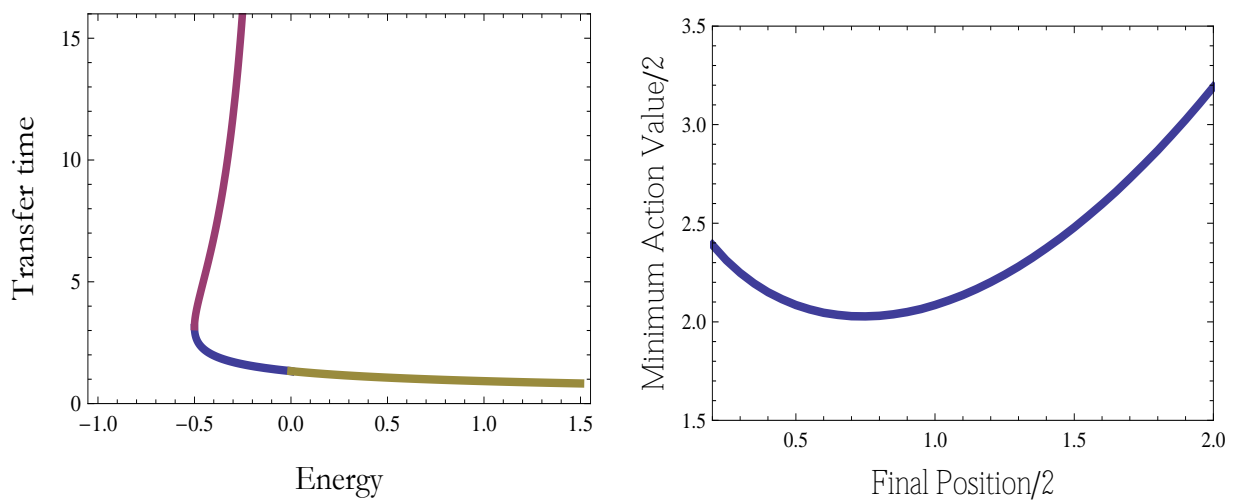

Figure 2: Left: Transfer time $T_{1}(0,2, \mathfrak{h})$ versus energy $\mathfrak{h}$. Right: Half of action value of ejection Keplerian orbit with final position $2 x$ and prescribed transfer time $T=2$.

\subsection{Minimization with one fixed and one variable endpoint}

Here we assume one fixed endpoint is $\xi \neq 0$ and the other endpoint varies along a ray emanating from the origin. It is sufficient to consider minimization over $\Gamma_{T}\left(\xi, \mathbb{R}_{+}\right)$or, equivalently, $\Gamma_{T}\left(\mathbb{R}_{+}, \xi\right)$. Define the infimum Keplerian action $\mathfrak{I}_{\lambda, T}: \mathbb{R}_{+} \times[0, \pi] \rightarrow \mathbb{R}_{+}$ by

$$
\mathfrak{I}_{\lambda, T}(\rho, \phi)=\inf \left\{I_{\lambda, T}(\mathbf{r}): \mathbf{r} \in \Gamma_{T}\left(\rho e^{i \phi}, \mathbb{R}_{+}\right)\right\}
$$

Or equivalently,

$$
\begin{aligned}
\mathfrak{I}_{\lambda, T}(\rho, \phi) & =\inf \left\{I_{\lambda, T}(\mathbf{r}): \mathbf{r} \in \Gamma_{T}\left(\rho, \mathbb{R}_{+} e^{i \phi}\right)\right\} \\
& =\inf \left\{I_{\lambda, T}(\mathbf{r}): \mathbf{r} \in \Gamma_{T}\left(\mathbb{R}_{+}, \rho e^{i \phi}\right)\right\} .
\end{aligned}
$$

Proposition 2.4. Given $\xi \neq 0$, let $\phi=\operatorname{Arg}(\xi)$. Consider the Keplerian action functional $I_{\lambda, T}$ restricted to $\Gamma_{T}\left(\mathbb{R}_{+}, \xi\right)$.

(a) If $\phi \in[0, \pi / 2]$, then for any action minimizer $\mathbf{r}$ of $I_{\lambda, T}, \mathbf{r}$ is free from collisions on $[0, T]$ and $\dot{\mathbf{r}}(0) \perp \mathbb{R}_{+}$.

(b) If $\phi \in(\pi / 2, \pi]$, then

$$
\inf _{\Gamma_{T}\left(\mathbb{R}_{+, \xi}\right)} I_{\lambda, T}>\inf _{\Gamma_{T}\left(\mathbb{R}_{+},-\bar{\xi}\right)} I_{\lambda, T}
$$

Proof. The first part follows from a standard blow-up and deformation arguments (see for instances [8, Section 5], [18, Sections 7,9] and [31, Section 4.1]), outlined as follows.

Suppose an action minimizer on $\Gamma_{T}\left(\mathbb{R}_{+}, \xi\right)$ has a collision, then there is a parabolic ejection orbit $y$ along the ray $\mathbb{R}_{+} \xi$ such that it minimizes $I_{\lambda, T^{\prime}}$ on $\Gamma_{T^{\prime}}\left(\mathbb{R}_{+}, y\left(T^{\prime}\right)\right)$ for any 
$T^{\prime}>0$. This orbit $y$ is given by $y(t)=\omega t^{\frac{2}{3}} e^{i \phi}$, where $\omega=(9 \lambda / 2)^{\frac{1}{3}}$ is as in Proposition 2.3. Now we prove by contradiction via a suitable deformation $\tilde{y}$ from $y$ that reduces the action value.

Suppose $\phi \in[0, \pi / 2)$. Let the deformation $\tilde{y}$ be given by

$$
\tilde{y}(t)= \begin{cases}\omega\left(\cos \phi+i t^{\frac{2}{3}} \sin \phi\right) & \text { for } t \in[0,1] \\ y(t) & \text { for } t \in(1, \infty) .\end{cases}
$$

Then clearly $\tilde{y} \in \Gamma_{1}\left(\mathbb{R}_{+}, y(1)\right)$ has lower action value on $[0,1]$ than $y$. Suppose $\phi=\pi / 2$. Given an arbitrary $\tau>1$. Let $\tilde{y}$ be given by

$$
\tilde{y}(t)= \begin{cases}(\omega+1) t^{\frac{2}{3}} e^{i \phi} & \text { for } t \in[0,1] \\ y(t)+(\tilde{y}(1)-y(1))\left(\frac{\tau-t}{\tau-1}\right) & \text { for } t \in(1, \tau] \\ y(t) & \text { for } t \in(\tau, \infty) .\end{cases}
$$

Then $\tilde{y} \in \Gamma_{\tau}\left(\mathbb{R}_{+}, y(\tau)\right)$ and

$$
\int_{0}^{\tau}|\dot{y}|^{2}-|\dot{\tilde{y}}|^{2} d t=\int_{1}^{\tau}|\dot{y}|^{2}-|\dot{\tilde{y}}|^{2} d t=\mathcal{O}\left(\tau^{-\frac{1}{3}}\right) \quad \text { as } \tau \rightarrow \infty,
$$

whereas

$$
\int_{0}^{1} \frac{\lambda}{|y(t)|}-\frac{\lambda}{|\tilde{y}(t)|} d t
$$

is a positive constant independent of $\tau$. This implies that $I_{\lambda, \tau}(y)>I_{\lambda, \tau}(\tilde{y})$ for $\tau$ sufficiently large, which is again a contradiction. The perpendicular condition $\dot{\mathbf{r}}(0) \perp \mathbb{R}_{+}$at $t=0$ is simply the natural boundary condition for regular extremals.

For the second part of the proposition, observe that $\operatorname{Arg}(-\bar{\xi})=\pi-\phi \in[0, \pi / 2)$. Given a minimizer $\mathbf{r}=r e^{i \theta}$ on $\Gamma_{T}\left(\mathbb{R}_{+}, \xi\right)$. Consider the path $\tilde{\mathbf{r}}=\tilde{r} e^{i \tilde{\theta}}$ given by

$$
\tilde{r} \equiv r, \quad \tilde{\theta}=\frac{\pi-\phi}{\phi} \theta .
$$

Then $\tilde{\mathbf{r}} \in \Gamma_{T}\left(\mathbb{R}_{+},-\bar{\xi}\right)$. It is obvious from the polar form of the Keplerian action functional (2.2) that $I_{\lambda, T}(\tilde{\mathbf{r}})<I_{\lambda, T}(\mathbf{r})$.

As a preparation for the main theorem in this subsection, we recall that in classical theory of variational calculus, the first and second variations for a functional of the form

$$
I(x)=\int_{t_{0}}^{t_{1}} L(x, \dot{x}) d t, \quad x \in C^{1}\left(\left[t_{0}, t_{1}\right], \mathbb{R}^{d}\right),
$$


are, assuming smoothness of the Lagrangian $L$, obtained by evaluating

$$
\begin{aligned}
\frac{d}{d s} I(x+s h)= & \int_{t_{0}}^{t_{1}} \frac{\partial}{\partial x} L(x+s h, \dot{x}+s \dot{h}) h+\frac{\partial}{\partial \dot{x}} L(x+s h, \dot{x}+s \dot{h}) \dot{h} d t \\
\frac{d^{2}}{d s^{2}} I(x+s h)=\int_{t_{0}}^{t_{1}} & \frac{\partial^{2}}{\partial x^{2}} L(x+s h, \dot{x}+s \dot{h})(h, h)+2 \frac{\partial^{2}}{\partial x \partial \dot{x}} L(x+s h, \dot{x}+s \dot{h})(h, \dot{h}) \\
& +\frac{\partial^{2}}{\partial \dot{x}^{2}} L(x+s h, \dot{x}+s \dot{h})(\dot{h}, \dot{h}) d t .
\end{aligned}
$$

Second derivatives of the Lagrangian in above are bilinear forms on the space of admissible variations. One may replace the variation $x+\operatorname{sh}$ from $x$ by a one-parameter family of curves $\left\{x_{s}\right\}_{-\varepsilon<s<\varepsilon}$ which depends differently on $s$ with $x_{0}=x$. Namely,

$$
\begin{aligned}
\frac{d}{d s} I\left(x_{s}\right)= & \int_{t_{0}}^{t_{1}} \frac{\partial}{\partial x} L\left(x_{s}, \dot{x}_{s}\right) \frac{d x_{s}}{d s}+\frac{\partial}{\partial \dot{x}} L\left(x_{s}, \dot{x}_{s}\right) \frac{d \dot{x}_{s}}{d s} d t, \\
\frac{d^{2}}{d s^{2}} I\left(x_{s}\right)= & \int_{t_{0}}^{t_{1}} \frac{\partial^{2}}{\partial x^{2}} L\left(x_{s}, \dot{x}_{s}\right)\left(\frac{d x_{s}}{d s}, \frac{d x_{s}}{d s}\right)+2 \frac{\partial^{2}}{\partial x \partial \dot{x}} L\left(x_{s}, \dot{x}_{s}\right)\left(\frac{d x_{s}}{d s}, \frac{d \dot{x}_{s}}{d s}\right) \\
& \quad+\frac{\partial^{2}}{\partial \dot{x}^{2}} L\left(x_{s}, \dot{x}_{s}\right)\left(\frac{d \dot{x}_{s}}{d s}, \frac{d \dot{x}_{s}}{d s}\right)+\frac{\partial}{\partial x} L\left(x_{s}, \dot{x}_{s}\right) \frac{d^{2} x_{s}}{d s^{2}}+\frac{\partial}{\partial \dot{x}} L\left(x_{s}, \dot{x}_{s}\right) \frac{d^{2} \dot{x}_{s}}{d s^{2}} d t .
\end{aligned}
$$

Putting the second derivative in a more familiar form, via integration by parts, one has

$$
\begin{aligned}
\frac{d^{2}}{d s^{2}} I\left(x_{s}\right)=\int_{t_{0}}^{t_{1}} & \frac{\partial}{\partial x}\left\{\frac{\partial}{\partial x} L\left(x_{s}, \dot{x}_{s}\right)-\frac{d}{d t} \frac{\partial}{\partial \dot{x}} L\left(x_{s}, \dot{x}_{s}\right)\right\}\left(\frac{d x_{s}}{d s}, \frac{d x_{s}}{d s}\right) \\
+ & \left\{\frac{\partial}{\partial x} L\left(x_{s}, \dot{x}_{s}\right)-\frac{d}{d t} \frac{\partial}{\partial \dot{x}} L\left(x_{s}, \dot{x}_{s}\right)\right\} \frac{d^{2} x_{s}}{d s^{2}}+\frac{\partial^{2}}{\partial \dot{x}^{2}} L\left(x_{s}, \dot{x}_{s}\right)\left(\frac{d \dot{x}_{s}}{d s}, \frac{d \dot{x}_{s}}{d s}\right) d t \\
+ & {\left[\frac{\partial^{2}}{\partial x \partial \dot{x}} L\left(x_{s}, \dot{x}_{s}\right)\left(\frac{d x_{s}}{d s}, \frac{d x_{s}}{d s}\right)+\frac{\partial}{\partial \dot{x}} L\left(x_{s}, \dot{x}_{s}\right) \frac{d^{2} x_{s}}{d s^{2}}\right]_{t_{0}}^{t_{1}} }
\end{aligned}
$$

Terms inside braces $\{\cdot\}$ vanish if the curve $x_{s}$ happens to be an extremal of the functional I.

Theorem 2.1. Given $\lambda>0, T>0$. The infimum Keplerian action $\mathfrak{I}_{\lambda, T}(\rho, \phi)$ defined on $\mathbb{R}_{+} \times(0, \pi / 2]$ is convex in $\rho$ and monotone increasing in $\phi$. Moreover, for fixed $\rho$ the function $\mathfrak{I}_{\lambda, T}(\rho, \phi)$ is increasing in $\phi \in[0, \pi]$, and for fixed $\phi$ the function has unique global minimum at $r_{\lambda, T, \phi}^{\sharp}=\left(\lambda T^{2} / \phi^{2}\right)^{\frac{1}{3}}$.

Proof. The proof for monotonicity in $\phi$ is similar to Proposition 2.4(b). Fix $\rho>0,0 \leq \tilde{\phi}<$ $\phi \leq \pi$, and let $\xi=\rho e^{i \phi}, \tilde{\xi}=\rho e^{i \tilde{\phi}}$. Given a minimizer $\mathbf{r}=r e^{i \theta}$ on $\Gamma_{T}\left(\mathbb{R}_{+}, \tilde{\xi}\right)$. Consider the path $\tilde{\mathbf{r}}=\tilde{r} e^{i \tilde{\theta}}$ given by

$$
\tilde{r} \equiv r, \quad \tilde{\theta}=\frac{\tilde{\phi}}{\phi} \theta
$$


Then $\tilde{\mathbf{r}} \in \Gamma_{T}\left(\mathbb{R}_{+}, \tilde{\xi}\right)$. It follows from the polar form of the Keplerian action functional (2.2) that

$$
\mathfrak{I}_{\lambda, T}(\rho, \tilde{\phi}) \leq I_{\lambda, T}(\tilde{\mathbf{r}}) \leq I_{\lambda, T}(\mathbf{r})=\mathfrak{I}_{\lambda, T}(\rho, \phi) .
$$

Thus $\mathfrak{I}_{\lambda, T}(\rho, \phi)$ is increasing in $\phi$.

Fix $\rho>0, \phi \in(0, \pi / 2)$. Let $\tilde{\mathbf{r}}=\tilde{\mathbf{r}}_{\rho, \phi}$ be a minimizer of $I_{\lambda, T}$ on $\left.\Gamma_{T}\left(\rho, \mathbb{R}_{+} e^{i \phi}\right)\right\}$. Being an action minimizer, it is a Keplerian orbit. Since $\phi \in(0, \pi / 2)$, by Proposition 2.4 the minimizer $\tilde{\mathbf{r}}$ has no collision and so the natural boundary condition resulting from the first variation of $I_{\lambda, T}$ is $\dot{\tilde{\mathbf{r}}}(T) \perp \mathbb{R}_{+} e^{i \phi}$. This implies that $\tilde{\mathbf{r}}$ is a conic with pericentre (periapsis) distance $|\tilde{\mathbf{r}}(T)|$, and this uniquely determines the action minimizer. For $s \in(-\varepsilon, \varepsilon)$ with small $\varepsilon>0$, let $\mathbf{r}_{s}$ be the minimizer with $\mathbf{r}_{s}(0)=\rho+s$, which is also uniquely determined. By Proposition 2.4(a) it is collision-free, and so $\mathbf{r}_{s}(t)$ depends smoothly on $s$ and $t$ provided $\varepsilon$ is sufficiently small. Then $\mathbf{r}_{0}=\tilde{\mathbf{r}}_{\rho, \phi}$ and $\mathbf{r}_{s}(T)=\mathbf{r}_{0}(T)+g(s) e^{i \phi}$ for some smooth function $g$ satisfying $g(0)=0$. Minimality of $\mathbf{r}_{s}$ ensures validity of the same natural boundary condition $\dot{\mathbf{r}}_{S}(T) \perp \mathbb{R}_{+} e^{i \phi}$.

Since each $\mathbf{r}_{s}$ is an extremal of the functional $I_{\lambda, T}$, we deduce from (2.10) that

$$
\begin{aligned}
& \frac{\partial^{2}}{\partial \rho^{2}} \Im_{\lambda, T}(\rho, \phi)=\left.\frac{d^{2}}{d s^{2}}\right|_{s=0} I_{\lambda, T}\left(\mathbf{r}_{s}\right), \\
& \frac{d^{2}}{d s^{2}} I_{\lambda, T}\left(\mathbf{r}_{s}\right)=\int_{0}^{T}\left|\frac{d \dot{\mathbf{r}}_{s}}{d s}\right|^{2} d t+\left[\dot{\mathbf{r}}_{s} \cdot \frac{d^{2} \mathbf{r}_{s}}{d s^{2}}\right]_{0}^{T} .
\end{aligned}
$$

In the second identity, the second term on the right-side equals zero because

$$
\dot{\mathbf{r}}_{s}(T) \cdot \frac{d^{2} \mathbf{r}_{s}}{d s^{2}}(T)=0,
$$

due to the natural boundary condition, and

$$
\dot{\mathbf{r}}_{s}(0) \cdot \frac{d^{2} \mathbf{r}_{s}}{d s^{2}}(0)=0,
$$

from the construction of $\mathbf{r}_{s}$. The first term on the right-side is clearly nonnegative. We claim that it is strictly positive. If it were zero, we would have $\frac{d \dot{\mathbf{r}}_{s}}{d s}=0$ for (Lebesgue) almost every $t$, and then $\frac{d \mathbf{r}_{s}}{d s}(t)$ would be independent of $t$. But $\frac{d \mathbf{r}_{\mathbf{s}}}{d s}(0)=1$, implying that $\frac{d \mathbf{r}_{s}}{d s}(T)=1$ and

$$
g(s) e^{i \phi}=\mathbf{r}_{s}(T)-\mathbf{r}_{0}(T)=s .
$$

This is impossible whenever $s \neq 0$ since the term on the left is purely imaginary but the term on the right is real. This shows that $\mathfrak{I}_{\lambda}(\rho, \theta)$ is strictly convex in $\rho$. The case $\phi=\pi / 2$ follows by continuity of $\mathfrak{I}_{\lambda}(\rho, \phi)$. 


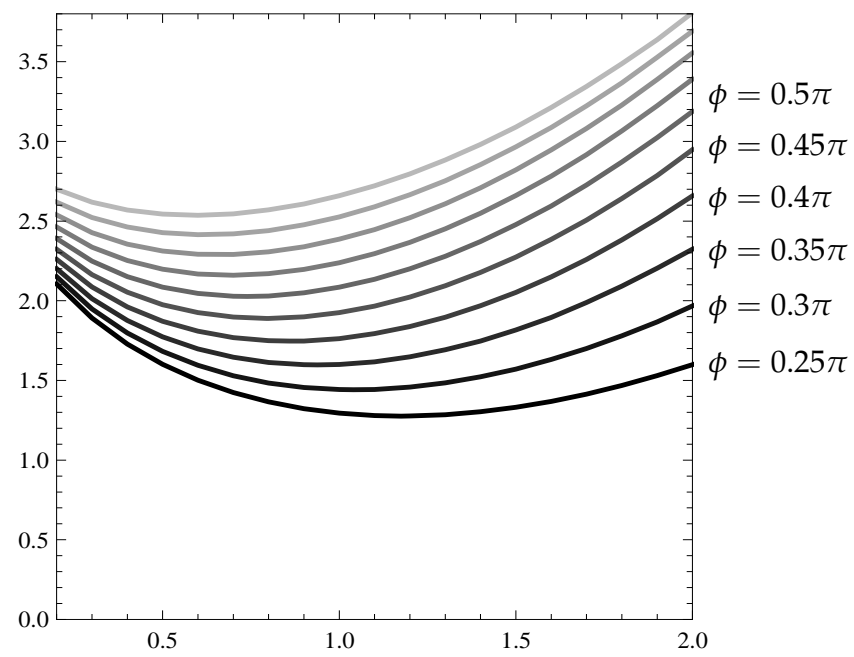

Figure 3: Graphs of $\mathfrak{I}_{1,1}(\rho, \phi)$ as a function of $\rho$.

Notation 2.1. Define $\mathfrak{E}_{\lambda, T}: \mathbb{R}_{+} \times(0, \pi) \rightarrow \mathbb{R}_{+}$by

$$
\mathfrak{E}_{\lambda, T}(\rho, \phi)=\mathfrak{I}_{\lambda, T}(\rho, \phi)-\frac{3}{2}(\lambda \phi)^{\frac{2}{3}} T^{\frac{1}{3}} .
$$

The special case $\mathfrak{E}_{1,1}$ is denoted by $\mathfrak{E}$.

By suitable scaling as did (2.3) we find

$$
\mathfrak{E}_{\lambda, T}(\rho, \phi)=\left(\lambda^{2} T\right)^{\frac{1}{3}} \mathfrak{E}\left(\left(\lambda T^{2}\right)^{-\frac{1}{3}} \rho, \phi\right) .
$$

As a simple corollary of Theorem 2.1 and Proposition 2.4, we have

Corollary 2.1. The function $\mathfrak{E}_{\lambda, T}(\rho, \phi)$ defined on $\mathbb{R}_{+} \times(0, \pi / 2]$ is nonnegative and convex in $\rho$. Moreover, for fixed $\phi$ it has unique global minimum at

$$
\rho=r_{\lambda, T, \phi}^{\sharp}=\left(\lambda T^{2} / \phi^{2}\right)^{\frac{1}{3}} \text { and } \mathfrak{E}_{\lambda, T}\left(r_{\lambda, T, \phi^{\prime}}^{\sharp} \phi\right)=0 \text {. }
$$

\section{Algebraic and topological classifications of planar motions}

Given $n$ mass points moving on the complex plane. Consider the boundary constraints that all mass points are confined to move from one line $L_{0}$ to another line $L_{1}$ in a given time $T$. Without loss of generality we let $L_{0}$ be the real axis and let $L_{1}=\mathbb{R} e^{\phi i}, \phi \in[0, \pi)$. The class of such paths is denoted by

$$
P_{\phi, T, n}:=\left\{x \in C\left([0, T], \mathbb{C}^{n}\right): x_{k}(0) \in \mathbb{R}, e^{-i \phi} x_{k}(T) \in \mathbb{R}, \forall k\right\} .
$$


Let $\Delta:=\left\{x \in \mathbb{C}^{n}: x_{i}=x_{j}\right.$ for some $\left.i \neq j\right\}$ be the variety of collision configurations. The subset

$$
P_{\phi, T, n}^{\times}:=\left\{x \in P_{\phi, T, n}: x(t) \in \mathbb{C}^{n} \backslash \Delta \text { for any } t \in[0, T]\right\}
$$

of $P_{\phi, T, n}$ consists of collision-free paths. In below we classify planar motions of $n$ bodies in $P_{\phi, T, n}^{\times}$by the symmetric group $S_{n}$ on $n$ symbols and the braid group $B_{n}$ on $n$ strands. Classifications using braids have been applied to planar motions in [26,27].

\subsection{Classification by the symmetric group $S_{n}$}

By relabeling indices if necessary, we may confine our path space to only those which begin with a prescribed ordering. Given $\sigma \in S_{n}$. Let

$$
P_{\phi, T, n}^{\sigma}:=\left\{x \in P_{\phi, T, n}^{\times}: \begin{array}{l}
x_{n}(0)<\cdots<x_{2}(0)<x_{1}(0) \\
e^{-i \phi} x_{\sigma_{n}}(T)<\cdots<e^{-i \phi} x_{\sigma_{2}}(T)<e^{-i \phi} x_{\sigma_{1}}(T)
\end{array}\right\} .
$$

In above $\sigma_{k}=\sigma(k)$. This provides an algebraic classification for planar motions in $P_{\phi, T, n}^{\times}$ by $S_{n}$.

Some people may prefer to replace $\sigma$ by $\sigma^{-1}$. We prefer our definition because it looks more natural to us and it is more compatible with multiplications of braid classes (see the next subsection). To make it more intuitive, one may interpret $\sigma_{k}$ as "where $k$-th mass comes from" and $\sigma_{k}^{-1}$ as "where $k$-th mass goes to". For example, $\sigma_{1}=2$ or $\sigma_{2}^{-1}=1$ means the second mass point goes to the first place, counting from right to left.

There are well-known solutions for the $n$-body problem passing through various collinear configurations, thereby providing examples of solutions within $P_{\phi, T, n}^{\sigma}$ for various $\phi$ and $\sigma$. What we are interested in here are those which perpendicular to their boundary lines, so that solution arcs can be extended indefinitely to globally defined relative periodic solutions by repeated reflections with respect to boundary lines. Such perpendicular conditions are exactly the natural boundary conditions for typical variational problems with varying endpoints. Whether or not an action minimizing solution exist in a given class $P_{\phi, T, n}^{\sigma}$ is our major task:

$$
\text { minimize } \mathcal{A}_{T} \text { over } P_{\phi, T, n}^{\sigma} \text {. }
$$

When the two boundary lines are transversal (i.e., $\phi \in(0, \pi)$ ), the action functional $\mathcal{A}_{T}$ is coercive on $P_{\phi, T, n}$, action minimizing orbits on $P_{\phi, T, n}$ exist and are free from collisions [8, Theorem 2.2]. Consequently, the action functional $\mathcal{A}_{T}$ attains its infimum on the weak closure of $P_{\phi, T, n}^{\sigma}$ for any $\sigma \in S_{n}$. When $\phi=0$, coercivity of $\mathcal{A}_{T}$ depends on the choice of $\sigma$.

Example 3.1. The simplest examples are the Euler-Moulton (collinear) relative equilibria with $n$ arbitrary masses. Given an arbitrary ordering of these masses. Let $x_{\mathrm{EM}}$ be such a relative equilibrium with period 1 and with mass center at the origin. Suppose $x_{\mathrm{EM}}$ 
rotates counterclockwise about the origin, then it belongs to $P_{\phi, \phi / 2 \pi, n}^{e}$ for any $\phi \in(0, \pi]$, where $e=()$ is the identity element of $S_{n}$. Let $\sigma$ be the order reversing permutation (i.e., $\sigma=(1 n)(2 n-1) \cdots)$. Then $x_{\mathrm{EM}}$ also belongs to $P_{\phi-\pi, \phi / 2 \pi, n}^{\sigma}$ for $\phi \in[\pi, 2 \pi]$. However, it is action minimizing on $P_{\phi, \phi / 2 \pi, n}^{e}$ for small $\phi$ but not on $P_{\phi-\pi, \phi / 2 \pi, n}^{\sigma}$ for small $\phi-\pi$ (see the next example).

Example 3.2. For fixed $T>0$, many retrograde orbits of the three-body problem are action minimizing on $P_{\phi, T, 3}^{\sigma}$ for $\sigma=(12)$ and for a large range of $\phi$ (see $\left.[9,10]\right)$. These are orbits with one pair of masses revolving around each other in one direction, their mass center and the other mass revolving around each other in the other direction. There is a critical angle $\phi_{c} \in(0, \pi)$ such that action minimizers are Euler-Moulton relative equilibria when $\phi \in\left(0, \phi_{c}\right]$ but not for $\phi \in\left(\phi_{c}, \pi\right]$ (see [11, Theorem 2.2]). Numerical evidences in $[9,10]$ suggest that such absolute minimizers usually change from EulerMoulton relative equilibria to retrograde orbits with relative period $T$.

Example 3.3. The Figure-8 orbit [15] of the three-body problem passes across every collinear configuration but it is not action minimizing in our function space since it is never perpendicular to the line when its configuration turns collinear.

We remark here that the classification by $S_{n}$ applies to non-planar motions as well. One may include paths moving in $\mathbb{R}^{3} \cong \mathbb{C} \times \mathbb{R}$ from the subspace $\mathbb{R} \times\{0\}$ to the subspace $\mathbb{R} e^{\phi i} \times\{0\}$. It would be interesting to determine, with congruent boundary constraints, whether action minimizers in the spatial problem are actually planar orbits.

\subsection{Classification by the braid group $B_{n}$}

The braid group $B_{n}$ on $n$ strands generalizes the symmetric group $S_{n}$ on $n$ symbols in the sense that $S_{n}$ is isomorphic to the quotient group $B_{n} / P_{n}$, where $P_{n}$ is the normal subgroup of $B_{n}$ consisting of pure braids (i.e., braids with the same starting and ending positions). Elements in $B_{n}$ have simple geometric interpretations that are ideal for descriptions of periodic planar $n$-body motions without collision, as the trajectory of mass points over one period in the three-dimensional space-time looks like a pure braid on $n$ strands. In contrast, elements in $S_{n}$ "forget" how these braids twist and wind, only keeping track of the final ordering.

Fix $T>0, \phi \in[0, \pi)$, and consider the rotating frame which rotates the inertial frame about the origin with angular velocity $\phi / T$. Then any path in $P_{\phi, T, n}^{\times}$traces out a braid in the three-dimensional space-time. Two braids are considered equivalent if they are homotopic; i.e. one can be continuously deformed to the other among the set $P_{\phi, T, n}^{\times}$. This defines an equivalence relation on $P_{\phi, T, n}^{\times}$, and equivalence classes are called braid classes.

Within any two braid classes $\gamma_{0}, \gamma_{1}$ we may pick representatives $c_{0}$ in $\gamma_{0}, c_{1}$ in $\gamma_{1}$ such that $c_{0}(T)=c_{1}(0)$. The standard definition of braids multiplication

$$
\left(c_{0} \cdot c_{1}\right)(t)= \begin{cases}c_{0}(2 t), & t \in[0, T / 2], \\ c_{1}(2 t-T), & t \in(T / 2, T],\end{cases}
$$


induces a well-defined multiplication $\gamma_{0} \cdot \gamma_{1}$ and group structure for braid classes. The multiplicative identity is called the trivial braid class belonging to, which there is a EulerMoulton's relative equilibrium. The group of braid classes is exactly the braid group $B_{n}$ on $n$ strands. Due to its geometric nature, we refer the classification by $B_{n}$ a topological classification for planar motions.

For any $\sigma \in S_{n}$, the space $P_{\phi, T, n}^{\sigma}$ has infinitely many components, each of which is a braid class. Confining to any of these braid classes, we may ask whether an action minimizing solution exist in there. Minimizing properties of collision-ejection Keplerian orbits [20] suggests that we should consider elementary braids without much twist. Let $\mathbf{b}^{\sigma}$ be a braid class in $P_{\phi, T, n}^{\sigma}$, the minimization problem of our interests is:

$$
\operatorname{minimize} \mathcal{A}_{T} \text { over } \mathbf{b}^{\sigma} \text {. }
$$

Note that if $\gamma$ is a component of $P_{\phi, T, n}^{\sigma}$ and $\gamma^{\prime}$ is a component of $P_{\phi, T, n}^{\sigma^{\prime}}$, then $\gamma \cdot \gamma^{\prime}$ is the component of $P_{\phi, T, n}^{\sigma \sigma^{\prime}}$ consisting of paths that follow the braid $\gamma$ for $t \in[0, T / 2]$ and follow $\gamma^{\prime}$ for $t \in(T / 2, T]$.

Elementary braid types for the case of three bodies include trivial (Euler), retrograde, and prograde (direct) braids, for which we refer readers to $[9,10]$ and references therein. Several elementary braids for the case of four bodies were listed in Fig. 4. We exclude braids which are identical to some braids on the list after $180^{\circ}$ rotation about the time axis.

\section{The action functional for the $n$-body problem}

In this section we provide some lower bound estimates for the action functional $\mathcal{A}_{T}$ and show how they can be associated with certain constraint convex optimization problems.

Definition 4.1. Given $x=\left(x_{1}, \cdots, x_{n}\right) \in P_{\phi, T, n}$. Denote the relative position $x_{i}-x_{j}$ by $x_{i j}$. We classify subscript pairs $\{(i, j): 1 \leq i \neq j \leq n\},(i, j)$ and $(j, i)$ been considered equivalent, according to the behavior of $x_{i j}=x_{i}-x_{j}$ :

$$
\begin{aligned}
& \mathbf{\Delta}_{0,1}(x)=\left\{(i, j): i<j, x_{i j} \in \Gamma_{T, \phi}^{*}\right\}, \\
& \mathbf{\Delta}_{0,2}(x)=\left\{(i, j): i<j, x_{i j} \in \Gamma_{T, \pi-\phi}^{*}\right\}, \\
& \mathbf{\Delta}_{0}(x)=\mathbf{\Delta}_{0,1}(x) \cup \mathbf{\Delta}_{0,2}(x), \\
& \mathbf{\Delta}_{1}(x)=\left\{(i, j): i<j, x_{i j} \in \Gamma_{T, \phi} \backslash \Gamma_{T, \phi}^{*}\right\}, \\
& \mathbf{\Delta}_{2}(x)=\left\{(i, j): i<j, x_{i j} \in \Gamma_{T, \pi-\phi} \backslash \Gamma_{T, \pi-\phi}^{*}\right\} .
\end{aligned}
$$

For simplicity we use $\boldsymbol{\Delta}_{k}$ to denote $\boldsymbol{\Delta}_{k}(x)$ when the path $x$ is fixed. We call elements in $\boldsymbol{\Delta}_{0}$ colliding pairs, elements in $\boldsymbol{\Delta}_{1} \cup \boldsymbol{\Delta}_{0,1}$ order-preserving pairs, and elements in $\boldsymbol{\Delta}_{2} \cup \boldsymbol{\Delta}_{0,2}$ orderreversing pairs. The intersection $\mathbf{\Delta}_{0,1} \cap \boldsymbol{\Delta}_{0,2}$ is not necessarily empty since $x_{i j}$ may begin or end at 0 . 


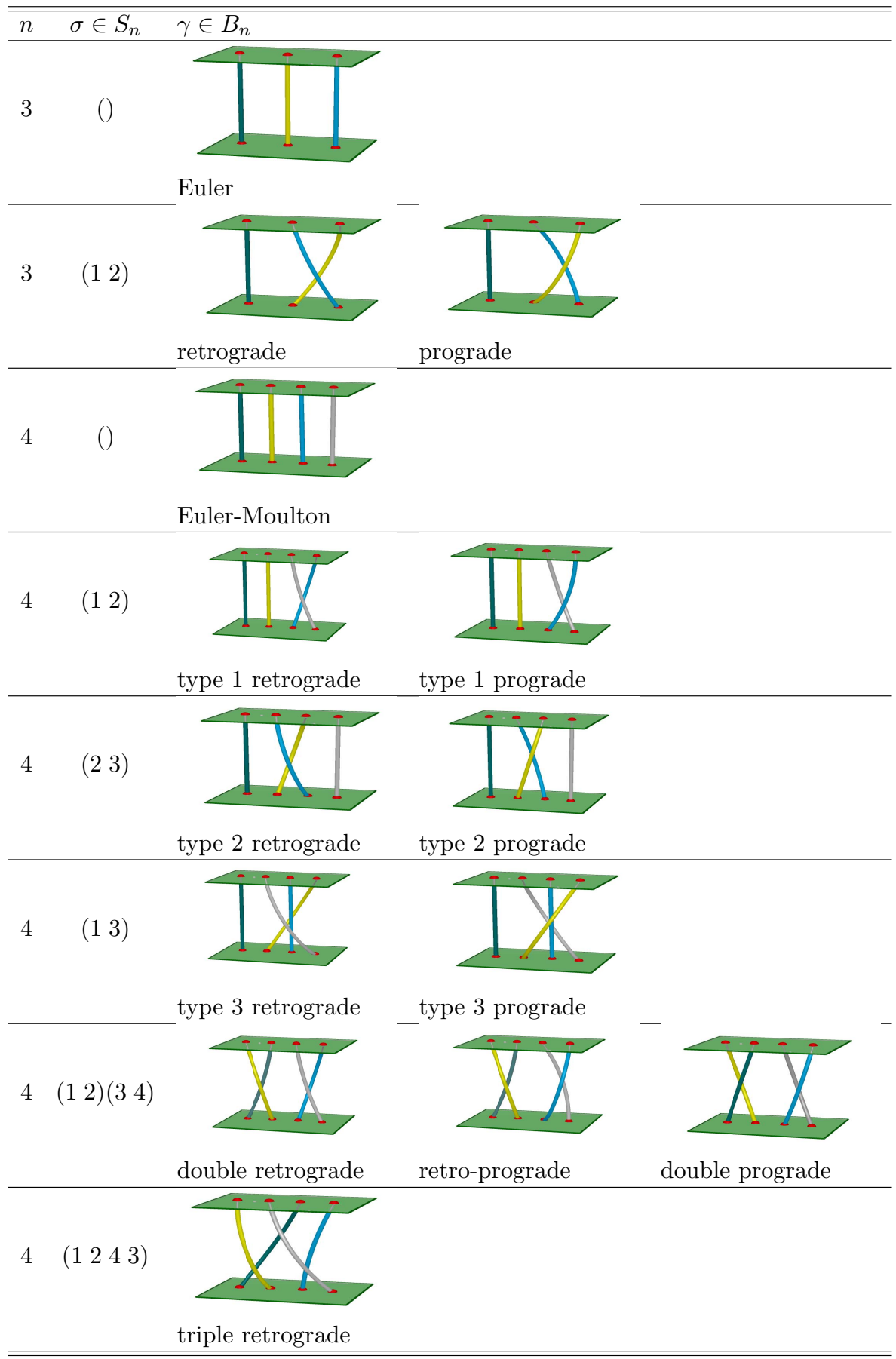

Figure 4: Some elements of $S_{n}$ and $B_{n}$. 
Clearly $\left\{\mathbf{\Delta}_{0}, \mathbf{\Delta}_{1}, \mathbf{\Delta}_{2}\right\}$ is a partition of subscript pairs. A partition $\left\{\mathbf{\Delta}_{0}^{\prime}, \mathbf{\Delta}_{1}^{\prime}, \mathbf{\Delta}_{2}^{\prime}\right\}$ of subscript pairs is called an ordered partition if

$$
\mathbf{\Delta}_{0}^{\prime} \subset \mathbf{\Delta}_{0}, \quad \mathbf{\Delta}_{1}^{\prime} \subset \mathbf{\Delta}_{1} \cup \mathbf{\Delta}_{0,1}, \quad \mathbf{\Delta}_{2}^{\prime} \subset \mathbf{\Delta}_{2} \cup \mathbf{\Delta}_{0,2} .
$$

That is, $\mathbf{\Delta}_{0}^{\prime}$ consists of colliding pairs, $\mathbf{\Delta}_{1}^{\prime}$ consists of order-preserving pairs, and $\mathbf{\Delta}_{2}^{\prime}$ consists of order-reversing pairs. The special case $\left\{\mathbf{\Delta}_{0}, \mathbf{\Delta}_{1}, \mathbf{\Delta}_{2}\right\}$ is called the standard ordered partition of subscript pairs.

Here is the main theorem of this section.

Theorem 4.1. Given $x \in P_{\phi, T, n}, \phi \in(0, \pi / 2]$. Let $M$ be the total mass, $\left\{\mathbf{\Delta}_{0}^{\prime}, \mathbf{\Delta}_{1}^{\prime}, \mathbf{\Delta}_{2}^{\prime}\right\}$ be any ordered partition of subscript pairs, and $\mathbb{E}$ be as in (2.11). Then

$$
\mathcal{A}_{T}(x) \geq\left(\frac{T}{M}\right)^{\frac{1}{3}} \max \left\{\mathfrak{A}_{0}, \mathfrak{A}_{T}, \mathfrak{B}_{0}, \mathfrak{B}_{T}\right\},
$$

where $\mathfrak{A}_{\tau}$ and $\mathfrak{B}_{\tau}, \tau \in\{0, T\}$, are given by

$$
\begin{aligned}
\mathfrak{A}_{\tau}= & \frac{3}{2}\left[\sum_{(i, j) \in \mathbf{\Lambda}_{0}^{\prime}} m_{i} m_{j} \pi^{\frac{2}{3}}+\sum_{(i, j) \in \mathbf{\Lambda}_{1}^{\prime}} m_{i} m_{j} \phi^{\frac{2}{3}}+\sum_{(i, j) \in \mathbf{\Lambda}_{2}^{\prime}} m_{i} m_{j}(\pi-\phi)^{\frac{2}{3}}\right] \\
& +\sum_{(i, j) \in \mathbf{\Lambda}_{1}^{\prime}} m_{i} m_{j} \mathfrak{E}\left(\left(M T^{2}\right)^{-\frac{1}{3}}\left|x_{i j}(\tau)\right|, \phi\right)+\sum_{(i, j) \in \mathbf{\Lambda}_{2}^{\prime}} m_{i} m_{j} \mathfrak{E}\left(\left(M T^{2}\right)^{-\frac{1}{3}}\left|x_{i j}(\tau)\right|, \pi-\phi\right), \\
\mathfrak{B}_{\tau}= & \frac{3}{2}\left[\sum_{(i, j) \in \mathbf{\Delta}_{0}^{\prime}} m_{i} m_{j} \pi^{\frac{2}{3}}+\sum_{(i, j) \in \mathbf{\Delta}_{1}^{\prime} \cup \mathbf{\Lambda}_{2}^{\prime}} m_{i} m_{j} \phi^{\frac{2}{3}}\right]+\sum_{(i, j) \in \mathbf{\Delta}_{1}^{\prime} \cup \mathbf{\Lambda}_{2}^{\prime}} m_{i} m_{j} \mathfrak{E}\left(\left(M T^{2}\right)^{-\frac{1}{3}}\left|x_{i j}(\tau)\right|, \phi\right) .
\end{aligned}
$$

Proof. Let $\hat{x}=\frac{1}{M} \sum_{k=1}^{N} m_{k} x_{k}$ be the mass center. The kinetic energy can be written

$$
K(\dot{x})=\frac{1}{2 M} \sum_{i<j} m_{i} m_{j}\left|\dot{x}_{i}-\dot{x}_{j}\right|^{2}+\frac{M}{2}|\dot{\hat{x}}|^{2} .
$$

Therefore, given $x \in P_{\phi, T, n}$,

$$
\begin{aligned}
\mathcal{A}_{T}(x) & \geq \frac{1}{M} \sum_{i<j} m_{i} m_{j} \int_{0}^{T} \frac{1}{2}\left|\dot{x}_{i}-\dot{x}_{j}\right|^{2}+\frac{M}{\left|x_{i}-x_{j}\right|} d t \\
& =\frac{1}{M} \sum_{i<j} m_{i} m_{j} I_{M, T}\left(x_{i j}\right) \\
& =\frac{1}{M}\left[\sum_{(i, j) \in \mathbf{\Lambda}_{0}^{\prime}}+\sum_{(i, j) \in \mathbf{\Lambda}_{1}^{\prime}}+\sum_{(i, j) \in \mathbf{\Lambda}_{2}^{\prime}} m_{i} m_{j} I_{M, T}\left(x_{i j}\right)\right] .
\end{aligned}
$$


If $(i, j) \in \mathbf{\Lambda}_{0}^{\prime}$, then by Proposition 2.1,

$$
I_{M, T}\left(x_{i j}\right) \geq \frac{3}{2}(M \pi)^{\frac{2}{3}} T^{\frac{1}{3}}
$$

If $(i, j) \in \mathbf{\Delta}_{1}^{\prime}$, then

$$
\begin{aligned}
& I_{M, T}\left(x_{i j}\right) \geq \mathfrak{I}_{M, T}\left(\left|x_{i j}(0)\right|, \phi\right) \\
= & \frac{3}{2}(M \phi)^{\frac{2}{3}} T^{\frac{1}{3}}+\mathfrak{E}_{M, T}\left(\left|x_{i j}(0)\right|, \phi\right) .
\end{aligned}
$$

If $(i, j) \in \mathbf{\Delta}_{2}^{\prime}$, then

$$
\begin{aligned}
& I_{M, T}\left(x_{i j}\right) \geq \mathfrak{I}_{M, T}\left(\left|x_{i j}(0)\right|, \pi-\phi\right) \\
= & \frac{3}{2}(M(\pi-\phi))^{\frac{2}{3}} T^{\frac{1}{3}}+\mathfrak{E}_{M, T}\left(\left|x_{i j}(0)\right|, \pi-\phi\right) .
\end{aligned}
$$

Summing up, we find

$$
\begin{aligned}
\mathcal{A}_{T}(x) \geq & \frac{3}{2}\left(\frac{T}{M}\right)^{\frac{1}{3}}\left[\sum_{(i, j) \in \mathbf{\Lambda}_{0}^{\prime}} m_{i} m_{j} \pi^{\frac{2}{3}}+\sum_{(i, j) \in \mathbf{\Lambda}_{1}^{\prime}} m_{i} m_{j} \phi^{\frac{2}{3}}+\sum_{(i, j) \in \mathbf{\Lambda}_{2}^{\prime}} m_{i} m_{j}(\pi-\phi)^{\frac{2}{3}}\right] \\
& +\frac{1}{M}\left[\sum_{(i, j) \in \mathbf{\Lambda}_{1}^{\prime}} m_{i} m_{j} \mathfrak{E}_{M, T}\left(\left|x_{i j}(0)\right|, \phi\right) .+\sum_{(i, j) \in \mathbf{\Lambda}_{2}^{\prime}} m_{i} m_{j} \mathfrak{E}_{M, T}\left(\left|x_{i j}(0)\right|, \pi-\phi\right)\right] .
\end{aligned}
$$

By the homogeneity property (2.12) of $\mathfrak{E}_{M, T}(\rho, \phi)$, we conclude that

$$
\mathcal{A}_{T}(x) \geq\left(\frac{T}{M}\right)^{\frac{1}{3}} \mathfrak{A}_{0} \text {. }
$$

The proof for

$$
\mathcal{A}_{T}(x) \geq\left(\frac{T}{M}\right)^{\frac{1}{3}} \mathfrak{A}_{T}
$$

is similar. Using an alternative definition for $\mathfrak{I}_{M, T}$ (see Subsection 2.3), the only observations needed here are

$$
\begin{array}{ll}
I_{M, T}\left(x_{i j}\right) \geq \mathfrak{I}_{M, T}\left(\left|x_{i j}(T)\right|, \phi\right) & \text { if }(i, j) \in \mathbf{\Delta}_{1}^{\prime}, \\
I_{M, T}\left(x_{i j}\right) \geq \mathfrak{I}_{M, T}\left(\left|x_{i j}(T)\right|, \pi-\phi\right) & \text { if }(i, j) \in \mathbf{\Delta}_{2}^{\prime} .
\end{array}
$$

The rest of the proof for this case is the same as the case $\tau=0$.

By Theorem 2.1 (or Proposition 2.4(b)), when $(i, j) \in \mathbf{\Delta}_{2}^{\prime}$, the Keplerian action $I_{M, T}\left(x_{i j}\right)$ can be estimated differently:

$$
\begin{aligned}
I_{M, T}\left(x_{i j}\right) & \geq \mathfrak{I}_{M, T}\left(\left|x_{i j}(0)\right|, \pi-\phi\right) \\
& \geq \mathfrak{I}_{M, T}\left(\left|x_{i j}(0)\right|, \phi\right) \\
& =\frac{3}{2}(M \phi)^{\frac{2}{3}} T^{\frac{1}{3}}+\mathfrak{E}_{M, T}\left(\left|x_{i j}(0)\right|, \phi\right) .
\end{aligned}
$$


With this estimate instead, one easily obtains

$$
\mathcal{A}_{T}(x) \geq\left(\frac{T}{M}\right)^{\frac{1}{3}} \mathfrak{B}_{0}
$$

The proof for $\mathcal{A}_{T}(x) \geq\left(\frac{T}{M}\right)^{\frac{1}{3}} \mathfrak{B}_{T}$ is similar.

Now we briefly explain how Theorem 4.1 is associated with convex optimization problems and how it works for $n$-body problems. Details are to be carried out in later sections.

Given positive masses $\left(m_{1}, \cdots, m_{n}\right), \phi \in(0, \pi / 2], T>0$, and $\sigma \in S_{n}$. There exist classical solutions for (1.1) in $P_{\phi, T, n}^{\sigma}$ as soon as we prove

$$
\inf _{P_{\phi, T, n}^{\sigma}} \mathcal{A}_{T}<\inf _{\partial P_{\phi, T, n}^{\sigma}} \mathcal{A}_{T} .
$$

Furthermore, if $\mathbf{b}^{\sigma}$ is a component of $P_{\phi, T, n}^{\sigma}$, then there exist classical solutions for (1.1) in the braid class $\mathbf{b}^{\sigma}$ if we have

$$
\inf _{\mathbf{b}^{\sigma}} \mathcal{A}_{T}<\inf _{\partial P_{\phi, T, n}^{\sigma}} \mathcal{A}_{T}
$$

The main difficulty is to provide good lower bound estimates for the right-hand side; i.e., action value of paths with collisions.

Note that for $x \in P_{\phi, T, n}^{\sigma}$ (which must be collision-free), order-preserving pairs and order reversing pairs are easily determined by $\sigma$ :

$$
\begin{aligned}
& \left\{(i, j): i<j, \sigma_{i}^{-1}<\sigma_{j}^{-1}\right\}=\mathbf{\Delta}_{1}, \\
& \left\{(i, j): i<j, \sigma_{i}^{-1}>\sigma_{j}^{-1}\right\}=\mathbf{\Delta}_{2} .
\end{aligned}
$$

If $x \in \partial P_{\phi, T, n^{\prime}}^{\sigma}(k, \ell) \in \mathbf{\Delta}_{0}$, we may select an ordered partition $\left\{\mathbf{\Delta}_{0}^{\prime}, \mathbf{\Lambda}_{1}^{\prime}, \mathbf{\Delta}_{2}^{\prime}\right\}$ by setting

$$
\begin{aligned}
& \mathbf{\Delta}_{0}^{\prime}=\{(k, \ell)\}, \\
& \mathbf{\Delta}_{1}^{\prime}=\left\{(i, j): i<j, \sigma_{i}^{-1}<\sigma_{j}^{-1},(i, j) \neq(k, \ell)\right\}, \\
& \mathbf{\Delta}_{2}^{\prime}=\left\{(i, j): i<j, \sigma_{i}^{-1}>\sigma_{j}^{-1},(i, j) \neq(k, \ell)\right\} .
\end{aligned}
$$

Then summations in $\mathfrak{A}_{\tau}$ and $\mathfrak{B}_{\tau}$ can be expressed in terms $\sigma$.

Taking the standard ordered partition for subscript pairs and dropping terms involving $\mathfrak{E}$, the inequality (4.2) clearly implies

$$
\mathcal{A}_{T}(x) \geq \frac{3}{2}\left(\frac{T}{M}\right)^{\frac{1}{3}}\left[\sum_{(i, j) \in \mathbf{\Delta}_{0}} m_{i} m_{j} \pi^{\frac{2}{3}}+\sum_{(i, j) \in \mathbf{\Delta}_{1}} m_{i} m_{j} \phi^{\frac{2}{3}}+\sum_{(i, j) \in \mathbf{\Delta}_{2}} m_{i} m_{j}(\pi-\phi)^{\frac{2}{3}}\right] .
$$


This is the lower bound estimate for the action functional one would obtained by following ideas in $[9,10]$. However, this lower bound estimate is not sufficient to extend results in $[9,10]$ to $n$-body problems with $n \geq 4$ and with general masses.

Theorem 4.1 is a very substantial improvement, especially when $n \geq 4$, since the contribution of summations involving $\mathfrak{E}$ can be quite considerable. While estimating either $\mathfrak{A}_{\tau}$ or $\mathfrak{B}_{\tau}$, we wish to find a definite lower bound for summations involving $\mathfrak{E}$ that is valid for all possible values of mutual distances $\left|x_{i j}(\tau)\right|$, which are therefore treated as variables over which the summation is minimized. Apparently, mutual distances are not independent variables. Minimizing summations involving $\mathfrak{E}$ with respect to mutual distances is a problem of convex optimization subject to constraints on the initial and final configurations. Simple techniques of convex analysis can be used to provide good lower bound estimates for this summation, as to be illustrated in the next section.

\section{Some constraint convex optimization problems}

Throughout this section we let $f: \mathbb{R}_{+} \rightarrow \mathbb{R}_{+}$be a nonnegative convex function with a global minimum at $u_{0}>0$. Consider the convex optimization problem of the form:

$$
\begin{cases}\text { minimize } & \sum_{k=1}^{m} \alpha_{k} f\left(u_{k}\right), \quad \alpha_{1}, \cdots, \alpha_{m} \text { are positive constants, } \\ \text { subject to } & A \mathbf{u}=0, \quad A \in \mathbb{R}^{p \times m}\end{cases}
$$

Here $\mathbf{u}=\left(u_{1}, \cdots, u_{m}\right)^{T}$.

A variant of this convex optimization problem considers an additional nonnegative convex function $g: \mathbb{R}_{+} \rightarrow \mathbb{R}_{+}$with a global minimum at $v_{0} \leq u_{0}$. The second type of optimization problem is of the form:

$$
\begin{cases}\text { minimize } & \sum_{k=1}^{\ell} \alpha_{k} f\left(u_{k}\right)+\sum_{k=\ell}^{m} \alpha_{k} g\left(u_{k}\right), \quad \alpha_{1}, \cdots, \alpha_{m} \text { are positive constants, } \\ \text { subject to } \quad A \mathbf{u} \leq 0, & A \in \mathbb{R}^{p \times m} .\end{cases}
$$

Here $A \mathbf{u} \leq 0$ means each of its components is non-positive.

The optimization problem of the form (5.1) will be applied to estimation of $\mathfrak{B}_{\tau}$, and (5.2) will be applied to estimation of $\mathfrak{A}_{\tau}$ in Theorem 4.1.

\subsection{The constraint convex optimization problem (5.1)}

The propositions below provide examples of lower bound estimates for such constraint convex optimization problems. 
Proposition 5.1. Consider the convex optimization problem (5.1) with $m=3, A=(1,1,-1)$. Then

$$
\sum_{k=1}^{3} \alpha_{k} f\left(u_{k}\right) \geq \min \left\{\alpha_{1} f\left(\bar{u}_{1}\right), \alpha_{2} f\left(\bar{u}_{2}\right), \alpha_{3} f\left(\bar{u}_{1}+\bar{u}_{2}\right)\right\}
$$

where

$$
\bar{u}_{1}=\frac{\alpha_{1}\left(\alpha_{2}+\alpha_{3}\right) u_{0}}{\alpha_{1} \alpha_{2}+\alpha_{1} \alpha_{3}+\alpha_{2} \alpha_{3}}, \quad \bar{u}_{2}=\frac{\alpha_{2}\left(\alpha_{1}+\alpha_{3}\right) u_{0}}{\alpha_{1} \alpha_{2}+\alpha_{1} \alpha_{3}+\alpha_{2} \alpha_{3}} .
$$

Proof. When $u_{1} \leq \bar{u}_{1}$, it follows from the convexity of $f$ and $\bar{u}_{1}<u_{0}$ that $\alpha_{1} f\left(\bar{u}_{1}\right)$ is a lower bound for the sum $\alpha_{1} f\left(u_{1}\right)+\alpha_{2} f\left(u_{2}\right)+\alpha_{3} f\left(u_{3}\right)$. Likewise, when $u_{2} \leq \bar{u}_{2}, \alpha_{2} f\left(\bar{u}_{2}\right)$ is a lower bound for the sum. When neither conditions hold, $u_{3}=u_{1}+u_{2}>\bar{u}_{1}+\bar{u}_{2}$, then by the convexity of $f$ and $\bar{u}_{1}+\bar{u}_{2}>u_{0}$, the summation is bounded from below by $\alpha_{3} f\left(\bar{u}_{1}+\bar{u}_{2}\right)$.

Proposition 5.2. Consider the convex optimization problem (5.1) with $m=5$. Suppose $\alpha_{1} \alpha_{4}=$ $\alpha_{2} \alpha_{3}$.

(a) If

$$
A=\left(\begin{array}{rrrrr}
1 & 0 & -1 & 0 & 1 \\
0 & 1 & 0 & -1 & 1
\end{array}\right)
$$

then

$$
\sum_{k=1}^{5} \alpha_{k} f\left(u_{k}\right) \geq \min \left\{\left(\alpha_{1}+\alpha_{2}\right) f\left(\bar{u}_{1}\right), \alpha_{5} f\left(\bar{u}_{2}\right),\left(\alpha_{3}+\alpha_{4}\right) f\left(\bar{u}_{1}+\bar{u}_{2}\right)\right\}
$$

where

$$
\begin{aligned}
& \bar{u}_{1}=\frac{\left(\alpha_{1}+\alpha_{2}\right)\left(\alpha_{3}+\alpha_{4}+\alpha_{5}\right) u_{0}}{\left(\alpha_{1}+\alpha_{2}\right)\left(\alpha_{3}+\alpha_{4}+\alpha_{5}\right)+\left(\alpha_{3}+\alpha_{4}\right) \alpha_{5}}, \\
& \bar{u}_{2}=\frac{\left(\alpha_{1}+\alpha_{2}+\alpha_{3}+\alpha_{4}\right) \alpha_{5} u_{0}}{\left(\alpha_{1}+\alpha_{2}+\alpha_{3}+\alpha_{4}\right) \alpha_{5}+\left(\alpha_{1}+\alpha_{2}\right)\left(\alpha_{3}+\alpha_{4}\right)} .
\end{aligned}
$$

(b) If

$$
A=\left(\begin{array}{lllll}
1 & 0 & 1 & 0 & -1 \\
0 & 1 & 0 & 1 & -1
\end{array}\right)
$$

then

$$
\sum_{k=1}^{5} \alpha_{k} f\left(u_{k}\right) \geq \min \left\{\left(\alpha_{1}+\alpha_{2}\right) f\left(\bar{u}_{1}\right),\left(\alpha_{3}+\alpha_{4}\right) f\left(\bar{u}_{2}\right), \alpha_{5} f\left(\bar{u}_{1}+\bar{u}_{2}\right)\right\},
$$

where

$$
\begin{aligned}
& \bar{u}_{1}=\frac{\left(\alpha_{1}+\alpha_{2}\right)\left(\alpha_{3}+\alpha_{4}+\alpha_{5}\right) u_{0}}{\left(\alpha_{1}+\alpha_{2}\right)\left(\alpha_{3}+\alpha_{4}+\alpha_{5}\right)+\left(\alpha_{3}+\alpha_{4}\right) \alpha_{5}}, \\
& \bar{u}_{2}=\frac{\left(\alpha_{3}+\alpha_{4}\right)\left(\alpha_{1}+\alpha_{2}+\alpha_{5}\right) u_{0}}{\left(\alpha_{3}+\alpha_{4}\right)\left(\alpha_{1}+\alpha_{2}+\alpha_{5}\right)+\left(\alpha_{1}+\alpha_{2}\right) \alpha_{5}} .
\end{aligned}
$$


Proof. By convexity,

$$
\sum_{k=1}^{5} \alpha_{k} f\left(u_{k}\right) \geq\left(\alpha_{1}+\alpha_{2}\right) f\left(\frac{\alpha_{1} u_{1}+\alpha_{2} u_{2}}{\alpha_{1}+\alpha_{2}}\right)+\left(\alpha_{3}+\alpha_{4}\right) f\left(\frac{\alpha_{3} u_{3}+\alpha_{4} u_{4}}{\alpha_{3}+\alpha_{4}}\right)+\alpha_{5} f\left(u_{5}\right) .
$$

Since $\alpha_{1} \alpha_{4}=\alpha_{2} \alpha_{3}$, the choice of $A$ implies

$$
\frac{\alpha_{1} u_{1}+\alpha_{2} u_{2}}{\alpha_{1}+\alpha_{2}}+u_{5}=\frac{\alpha_{3} u_{3}+\alpha_{4} u_{4}}{\alpha_{3}+\alpha_{4}} .
$$

Now part (a) follows easily from Proposition 5.1(a), with $\left(\alpha_{1}, \alpha_{2}, \alpha_{3}\right)$ and $\left(u_{1}, u_{2}, u_{3}\right)$ in Proposition 5.1(a) replaced respectively by $\left(\alpha_{1}+\alpha_{2}, \alpha_{5}, \alpha_{3}+\alpha_{4}\right)$ and $\left(\frac{\alpha_{1} u_{1}+\alpha_{2} u_{2}}{\alpha_{1}+\alpha_{2}}, u_{5}, \frac{\alpha_{3} u_{3}+\alpha_{4} u_{4}}{\alpha_{3}+\alpha_{4}}\right)$.

Part (b) is similar to (a). The difference is that now we have

$$
\frac{\alpha_{1} u_{1}+\alpha_{2} u_{2}}{\alpha_{1}+\alpha_{2}}-u_{5}=\frac{\alpha_{3} u_{3}+\alpha_{4} u_{4}}{\alpha_{3}+\alpha_{4}} .
$$

All we need is to replace $\left(\alpha_{1}, \alpha_{2}, \alpha_{3}\right)$ in Proposition 5.1 by $\left(\alpha_{1}+\alpha_{2}, \alpha_{3}+\alpha_{4}, \alpha_{5}\right)$, and replace $\left(u_{1}, u_{2}, u_{3}\right)$ in Proposition 5.1 by $\left(\frac{\alpha_{1} u_{1}+\alpha_{2} u_{2}}{\alpha_{1}+\alpha_{2}}, \frac{\alpha_{3} u_{3}+\alpha_{4} u_{4}}{\alpha_{3}+\alpha_{4}}, u_{5}\right)$.

Now we apply Propositions 5.1, 5.2 to estimate $\mathfrak{B}_{0}$ in (4.2) for the four-body problem.

Proposition 5.3. Given $\phi \in(0, \pi / 2]$ and $x \in P_{\phi, T, 4}$. Suppose

$$
x_{4}(0) \leq x_{3}(0) \leq x_{2}(0) \leq x_{1}(0) .
$$

Let $\mathfrak{E}$ be as in (2.11), $\left\{\boldsymbol{\Lambda}_{0}^{\prime}, \mathbf{\Delta}_{1}^{\prime}, \mathbf{\Delta}_{2}^{\prime}\right\}$ be an ordered partition of subscript pairs. Given positive masses $m_{i}, i \in\{1,2,3,4\}$. Let $M$ be the total mass, and

$$
\mathcal{E}_{0}=\sum_{(i, j) \in \mathbf{\Delta}_{1}^{\prime} \cup \mathbf{\Delta}_{2}^{\prime}} m_{i} m_{j} \mathfrak{E}\left(\left(M T^{2}\right)^{-\frac{1}{3}}\left|x_{i j}(0)\right|, \phi\right) .
$$

Then $\mathcal{E}_{0}$ has lower bounds given as follows.

(a) If $\mathbf{\Delta}_{0}^{\prime}=\{(1,2)\}$, then

$$
\mathcal{E}_{0} \geq\left(m_{1}+m_{2}\right) \min \left\{m_{3} \mathfrak{E}\left(\frac{M-m_{4}}{M \phi^{\frac{2}{3}}}, \phi\right), \frac{m_{3} m_{4}}{m_{1}+m_{2}} \mathfrak{E}\left(\frac{m_{3}+m_{4}}{M \phi^{\frac{2}{3}}}, \phi\right), m_{4} \mathfrak{E}\left(\frac{M+m_{3}}{M \phi^{\frac{2}{3}}}, \phi\right)\right\} .
$$

(b) If $\mathbf{\Delta}_{0}^{\prime}=\{(3,4)\}$, then

$$
\mathcal{E}_{0} \geq\left(m_{3}+m_{4}\right) \min \left\{m_{2} \mathfrak{E}\left(\frac{M-m_{1}}{M \phi^{\frac{2}{3}}}, \phi\right), \frac{m_{1} m_{2}}{m_{3}+m_{4}} \mathfrak{E}\left(\frac{m_{1}+m_{2}}{M \phi^{\frac{2}{3}}}, \phi\right), m_{1} \mathfrak{E}\left(\frac{M+m_{2}}{M \phi^{\frac{2}{3}}}, \phi\right)\right\} .
$$


(c) If $\mathbf{\Delta}_{0}^{\prime}=\{(2,3)\}$, then

$$
\mathcal{E}_{0} \geq\left(m_{2}+m_{3}\right) \min \left\{m_{1} \mathfrak{E}\left(\frac{M-m_{4}}{M \phi^{\frac{2}{3}}}, \phi\right), m_{4} \mathfrak{E}\left(\frac{M-m_{1}}{M \phi^{\frac{2}{3}}}, \phi\right), \frac{m_{1} m_{4}}{m_{2}+m_{3}} \mathfrak{E}\left(\frac{M+m_{2}+m_{3}}{M \phi^{\frac{2}{3}}}, \phi\right)\right\} .
$$

Proof. Fix $\phi \in(0, \pi / 2]$ and let

$$
f(u)=\mathfrak{E}\left(\left(M T^{2}\right)^{-\frac{1}{3}} u, \phi\right) .
$$

Then

$$
f(u)=\left(M^{2} T\right)^{-\frac{1}{3}} \mathfrak{E}_{M, T}(u, \phi),
$$

which has global minimum value 0 at $u_{0}=\left(M T^{2} / \phi^{2}\right)^{1 / 3}$, by Corollary 2.1.

When $\mathbf{\Delta}_{0}^{\prime}=\{(1,2)\}$, the lower bound in (a) is obtained by Proposition 5.2(a) with

$$
\begin{aligned}
& \left(u_{1}, u_{2}, u_{3}, u_{4}, u_{5}\right)=\left(x_{13}(0), x_{23}(0), x_{14}(0), x_{24}(0), x_{34}(0)\right), \\
& \left(\alpha_{1}, \alpha_{2}, \alpha_{3}, \alpha_{4}, \alpha_{5}\right)=\left(m_{1} m_{3}, m_{2} m_{3}, m_{1} m_{4}, m_{2} m_{4}, m_{3} m_{4}\right) .
\end{aligned}
$$

When $\boldsymbol{\Delta}_{0}^{\prime}=\{(3,4)\}$, the lower bound in (b) is obtained by Proposition 5.2(a) with

$$
\begin{aligned}
& \left(u_{1}, u_{2}, u_{3}, u_{4}, u_{5}\right)=\left(x_{23}(0), x_{24}(0), x_{13}(0), x_{14}(0), x_{12}(0)\right), \\
& \left(\alpha_{1}, \alpha_{2}, \alpha_{3}, \alpha_{4}, \alpha_{5}\right)=\left(m_{2} m_{3}, m_{2} m_{4}, m_{1} m_{3}, m_{1} m_{4}, m_{1} m_{2}\right) .
\end{aligned}
$$

When $\mathbf{\Delta}_{0}^{\prime}=\{(2,3)\}$, the lower bound in (c) is obtained by Proposition 5.2(b) with

$$
\begin{aligned}
& \left(u_{1}, u_{2}, u_{3}, u_{4}, u_{5}\right)=\left(x_{12}(0), x_{13}(0), x_{24}(0), x_{34}(0), x_{14}(0)\right), \\
& \left(\alpha_{1}, \alpha_{2}, \alpha_{3}, \alpha_{4}, \alpha_{5}\right)=\left(m_{1} m_{2}, m_{1} m_{3}, m_{2} m_{4}, m_{3} m_{4}, m_{1} m_{4}\right) .
\end{aligned}
$$

We complete the proof.

Remark 5.1. There are three cases involving single colliding pair that are missing in Proposition 5.3; i.e., the cases $\boldsymbol{\Delta}_{0}^{\prime}=\{(1,3)\},\{(2,4)\}$, and $\{(1,4)\}$. An estimate for the case $\{(1,4)\}$ can be easily obtained by applying Proposition 5.1 twice with

$$
\begin{aligned}
& \left(u_{1}, u_{2}, u_{3}\right)=\left(x_{12}(0), x_{23}(0), x_{13}(0)\right), \\
& \left(\alpha_{1}, \alpha_{2}, \alpha_{3}\right)=\left(m_{1} m_{2}, \frac{m_{1} m_{2}^{2} m_{3}}{m_{1} m_{2}+m_{3} m_{4}}, m_{1} m_{3}\right), \\
& \left(u_{1}, u_{2}, u_{3}\right)=\left(x_{34}(0), x_{23}(0), x_{24}(0)\right), \\
& \left(\alpha_{1}, \alpha_{2}, \alpha_{3}\right)=\left(m_{3} m_{4}, \frac{m_{2} m_{3}^{2} m_{4}}{m_{1} m_{2}+m_{3} m_{4}}, m_{2} m_{4}\right) .
\end{aligned}
$$

The other two cases can be estimated by imitating the proof for Proposition 5.1. We skip details here because they are not used in our applications. 
Similarly, one can obtain lower bound estimates for $\mathfrak{B}_{T}$ in (4.2):

Proposition 5.4. Given $\phi \in(0, \pi / 2], x \in P_{\phi, T, 4}$, and $\sigma \in S_{4}$. Suppose

$$
e^{-i \phi} x_{\sigma_{4}}(T) \leq e^{-i \phi} x_{\sigma_{3}}(T) \leq e^{-i \phi} x_{\sigma_{2}}(T) \leq e^{-i \phi} x_{\sigma_{1}}(T) \text {. }
$$

Let $\mathfrak{E}$ be as in (2.11), $\left\{\mathbf{\Delta}_{0}^{\prime}, \mathbf{\Delta}_{1}^{\prime}, \mathbf{\Delta}_{2}^{\prime}\right\}$ be an ordered partition of subscript pairs. Given positive masses $m_{i}, i \in\{1,2,3,4\}$. Let $M$ be the total mass, and

$$
\mathcal{E}_{T}=\sum_{(i, j) \in \mathbf{\Delta}_{1}^{\prime} \cup \mathbf{\Delta}_{2}^{\prime}} m_{i} m_{j} \mathfrak{E}\left(\left(M T^{2}\right)^{-\frac{1}{3}}\left|x_{i j}(T)\right|, \phi\right) .
$$

Then $\mathcal{E}_{T}$ has lower bounds given as follows.

(a) If $\mathbf{\Delta}_{0}^{\prime}=\left\{\left(\sigma_{1}, \sigma_{2}\right)\right\}$, then

$$
\begin{gathered}
\mathcal{E}_{T} \geq\left(m_{\sigma_{1}}+m_{\sigma_{2}}\right) \min \left\{m_{\sigma_{3}} \mathfrak{E}\left(\frac{M-m_{\sigma_{4}}}{M \phi^{\frac{2}{3}}}, \phi\right), \frac{m_{\sigma_{3}} m_{\sigma_{4}}}{m_{\sigma_{1}}+m_{\sigma_{2}}} \mathfrak{E}\left(\frac{m_{\sigma_{3}}+m_{\sigma_{4}}}{M \phi^{\frac{2}{3}}}, \phi\right),\right. \\
\left.m_{\sigma_{4}} \mathfrak{E}\left(\frac{M+m_{\sigma_{3}}}{M \phi^{\frac{2}{3}}}, \phi\right)\right\} .
\end{gathered}
$$

(b) If $\mathbf{\Delta}_{0}^{\prime}=\left\{\left(\sigma_{3}, \sigma_{4}\right)\right\}$, then

$$
\begin{gathered}
\mathcal{E}_{T} \geq\left(m_{\sigma_{3}}+m_{\sigma_{4}}\right) \min \left\{m_{\sigma_{2}} \mathfrak{E}\left(\frac{M-m_{\sigma_{1}}}{M \phi^{\frac{2}{3}}}, \phi\right), \frac{m_{\sigma_{1}} m_{\sigma_{2}}}{m_{\sigma_{3}}+m_{\sigma_{4}}} \mathfrak{E}\left(\frac{m_{\sigma_{1}}+m_{\sigma_{2}}}{M \phi^{\frac{2}{3}}}, \phi\right),\right. \\
\left.m_{\sigma_{1}} \mathfrak{E}\left(\frac{M+m_{\sigma_{2}}}{M \phi^{\frac{2}{3}}}, \phi\right)\right\} .
\end{gathered}
$$

(c) If $\mathbf{\Delta}_{0}^{\prime}=\left\{\left(\sigma_{2}, \sigma_{3}\right)\right\}$, then

$$
\begin{gathered}
\mathcal{E}_{T} \geq\left(m_{\sigma_{2}}+m_{\sigma_{3}}\right) \min \left\{m_{\sigma_{1}} \mathfrak{E}\left(\frac{M-m_{\sigma_{4}}}{M \phi^{\frac{2}{3}}}, \phi\right), m_{\sigma_{4}} \mathfrak{E}\left(\frac{M-m_{\sigma_{1}}}{M \phi^{\frac{2}{3}}}, \phi\right),\right. \\
\left.\frac{m_{\sigma_{1}} m_{\sigma_{4}}}{m_{\sigma_{2}}+m_{\sigma_{3}}} \mathfrak{E}\left(\frac{M+m_{\sigma_{2}}+m_{\sigma_{3}}}{M \phi^{\frac{2}{3}}}, \phi\right)\right\} .
\end{gathered}
$$

With each $m_{k}$ replaced by $m_{\sigma_{k}}$, the proof is exactly the same as the proof for Proposition 5.3.

For brevity, we introduce some notations to shorten expressions in Propositions 5.3 and 5.4. Given four positive masses $\left(m_{1}, m_{2}, m_{3}, m_{4}\right)$ and a fixed angle $\phi \in(0, \pi / 2]$. Let $M$ be the total mass and let $\{i, j, k, \ell\}=\{1,2,3,4\}$. Define

$$
\begin{aligned}
& E_{i j k \ell}=\left(m_{i}+m_{j}\right) \min \left\{m_{k} \mathfrak{E}\left(\frac{M-m_{\ell}}{M \phi^{\frac{2}{3}}}, \phi\right), \frac{m_{k} m_{\ell}}{m_{i}+m_{j}} \mathfrak{E}\left(\frac{m_{k}+m_{\ell}}{M \phi^{\frac{2}{3}}}, \phi\right), m_{\ell} \mathfrak{E}\left(\frac{M+m_{k}}{M \phi^{\frac{2}{3}}}, \phi\right)\right\}, \\
& F_{i j k \ell}=\left(m_{i}+m_{j}\right) \min \left\{m_{k} \mathfrak{E}\left(\frac{M-m_{\ell}}{M \phi^{\frac{2}{3}}}, \phi\right), m_{\ell} \mathfrak{E}\left(\frac{M-m_{k}}{M \phi^{\frac{2}{3}}}, \phi\right), \frac{m_{k} m_{\ell}}{m_{i}+m_{j}} \mathfrak{E}\left(\frac{M+m_{i}+m_{j}}{M \phi^{\frac{2}{3}}}, \phi\right)\right\} .
\end{aligned}
$$


Note that $E_{i j k \ell}=E_{j i k \ell}, F_{i j k \ell}=F_{j i k \ell}=F_{i j \ell k}=F_{j i \ell k}$. Under assumptions of Propositions 5.3 and 5.4, these two propositions state that

$$
\begin{array}{ll}
\mathbf{\Delta}_{0}^{\prime}=\{(1,2)\} & \text { implies } \mathcal{E}_{0} \geq E_{1234}, \\
\mathbf{\Delta}_{0}^{\prime}=\{(3,4)\} & \text { implies } \mathcal{E}_{0} \geq E_{4321}, \\
\mathbf{\Delta}_{0}^{\prime}=\{(2,3)\} & \text { implies } \mathcal{E}_{0} \geq F_{2314}, \\
\mathbf{\Delta}_{0}^{\prime}=\left\{\left(\sigma_{1}, \sigma_{2}\right)\right\} & \text { implies } \mathcal{E}_{T} \geq E_{\sigma_{1} \sigma_{2} \sigma_{3} \sigma_{4},} \\
\mathbf{\Delta}_{0}^{\prime}=\left\{\left(\sigma_{3}, \sigma_{4}\right)\right\} & \text { implies } \mathcal{E}_{T} \geq E_{\sigma_{4} \sigma_{3} \sigma_{2} \sigma_{1},} \\
\mathbf{\Delta}_{0}^{\prime}=\left\{\left(\sigma_{2}, \sigma_{3}\right)\right\} & \text { implies } \mathcal{E}_{T} \geq F_{\sigma_{2} \sigma_{3} \sigma_{1} \sigma_{4}} .
\end{array}
$$

We end this subsection with further improvements of Proposition 5.1, with which Propositions 5.2, 5.3, 5.4 can be improved accordingly.

Proposition 5.5. Consider the convex optimization problem (5.1) with $m=3, A=(1,1,-1)$. Fix $N \geq 3$. Let

$$
w_{i}= \begin{cases}\frac{(i+1) u_{0}}{N}, & \text { if } i<N, \\ \frac{i u_{0}}{N}, & \text { if } i \geq N .\end{cases}
$$

Then $\sum_{i=1}^{3} \alpha_{i} f\left(u_{i}\right)$ is bounded from below by

$$
\begin{array}{r}
\min \left\{\min _{j+k<N}\left\{\alpha_{1} f\left(w_{j}\right)+\alpha_{2} f\left(w_{k}\right)+\alpha_{3} f\left(w_{j+k+1}\right)\right\},\right. \\
\left.\min _{j+k \geq N}\left\{\alpha_{1} f\left(w_{j}\right)+\alpha_{2} f\left(w_{k}\right)+\alpha_{3} f\left(w_{j+k}\right)\right\}\right\} .
\end{array}
$$

Proof. Given $u_{1}, u_{2}>0$, there exists some $j, k \in\{0,1,2, \cdots\}$ such that

$$
\frac{j u_{0}}{N}<u_{1} \leq \frac{(j+1) u_{0}}{N}, \quad \frac{k u_{0}}{N}<u_{2} \leq \frac{(k+1) u_{0}}{N} .
$$

By convexity of $f$ and the assumption that $f$ has minimum at $u_{0}>0$, we always have

$$
f\left(u_{1}\right) \geq f\left(w_{j}\right), \quad f\left(u_{2}\right) \geq f\left(w_{k}\right) .
$$

If $j+k<N$, then $0<j, k<N$ and

$$
f\left(u_{1}+u_{2}\right) \geq \begin{cases}f\left(\frac{(j+k+2) u_{0}}{N}\right), & \text { if } j+k+1<N, \\ f\left(u_{0}\right), & \text { if } j+k+1=N .\end{cases}
$$

In either case, the right side can be written $f\left(w_{j+k+1}\right)$. Thus

$$
\alpha_{1} f\left(u_{1}\right)+\alpha_{2} f\left(u_{2}\right)+\alpha_{3} f\left(u_{1}+u_{2}\right) \geq \alpha_{1} f\left(w_{j}\right)+\alpha_{2} f\left(w_{k}\right)+\alpha_{3} f\left(w_{j+k+1}\right) .
$$


If $j+k \geq N$, then the convexity assumption on $f$ ensures that

$$
f\left(u_{1}+u_{2}\right) \geq f\left(\frac{(j+k) u_{0}}{N}\right)=f\left(w_{j+k}\right) .
$$

This implies the asserted inequality for the case $j+k \geq N$.

\subsection{The constraint convex optimization problem (5.2) with two convex functions}

We only consider a few cases related to our applications. Arguments in here are similar to those in the previous subsection. We begin with some analogues of Proposition 5.1.

Proposition 5.6. Consider the convex optimization problem (5.2) with $m=3, A=(1,1,-1)$.

(a) If $\ell=2$, then

$$
\alpha_{1} f\left(u_{1}\right)+\alpha_{2} f\left(u_{2}\right)+\alpha_{3} g\left(u_{3}\right) \geq \min \left\{\alpha_{1} f\left(\bar{u}_{1}\right), \alpha_{2} f\left(\bar{u}_{2}\right), \alpha_{3} g\left(\bar{u}_{1}+\bar{u}_{2}\right)\right\},
$$

where

$$
\bar{u}_{1}=\frac{\alpha_{1}\left(\alpha_{2} u_{0}+\alpha_{3} v_{0}\right)}{\alpha_{1} \alpha_{2}+\alpha_{1} \alpha_{3}+\alpha_{2} \alpha_{3}}, \quad \bar{u}_{2}=\frac{\alpha_{2}\left(\alpha_{1} u_{0}+\alpha_{3} v_{0}\right)}{\alpha_{1} \alpha_{2}+\alpha_{1} \alpha_{3}+\alpha_{2} \alpha_{3}} .
$$

(b) If $\ell=1$, then

$$
\alpha_{1} f\left(u_{1}\right)+\alpha_{2} g\left(u_{2}\right)+\alpha_{3} g\left(u_{3}\right) \geq \min \left\{\alpha_{1} f\left(\bar{u}_{1}\right), \alpha_{2} g\left(\bar{u}_{2}\right), \alpha_{3} g\left(\bar{u}_{1}+\bar{u}_{2}\right)\right\},
$$

where

$$
\bar{u}_{1}=\frac{\alpha_{1}\left(\alpha_{2} u_{0}+\alpha_{3} v_{0}\right)}{\alpha_{1} \alpha_{2}+\alpha_{1} \alpha_{3}+\alpha_{2} \alpha_{3}}, \quad \bar{u}_{2}=\frac{\alpha_{2}\left(\alpha_{1}+\alpha_{3}\right) v_{0}}{\alpha_{1} \alpha_{2}+\alpha_{1} \alpha_{3}+\alpha_{2} \alpha_{3}} .
$$

Proof. The proof for part (a) is almost identical to Proposition 5.1. The constraint is simply $u_{1}+u_{2} \leq u_{3}$. When $u_{1} \leq \bar{u}_{1}$, it follows from the convexity of $f$ and $\bar{u}_{1}<u_{0}$ that $\alpha_{1} f\left(\bar{u}_{1}\right)$ is a lower bound for the sum $\alpha_{1} f\left(u_{1}\right)+\alpha_{2} f\left(u_{2}\right)+\alpha_{3} g\left(u_{3}\right)$. Likewise, when $u_{2} \leq \bar{u}_{2}, \alpha_{2} f\left(\bar{u}_{2}\right)$ is a lower bound for the sum. When neither conditions hold, $u_{3} \geq u_{1}+u_{2}>\bar{u}_{1}+\bar{u}_{2}$, then by the convexity of $g$ and $\bar{u}_{1}+\bar{u}_{2}>v_{0}$, the summation is bounded from below by $\alpha_{3} g\left(\bar{u}_{1}+\bar{u}_{2}\right)$. This proves (a).

In part (b) we have $\bar{u}_{2}<v_{0}$. When $u_{1} \leq \bar{u}_{1}$, as in part (a) we see that $\alpha_{1} f\left(\bar{u}_{1}\right)$ is a lower bound for the sum $\alpha_{1} f\left(u_{1}\right)+\alpha_{2} g\left(u_{2}\right)+\alpha_{3} g\left(u_{3}\right)$. When $u_{2} \leq \bar{u}_{2}, \alpha_{2} g\left(\bar{u}_{2}\right)$ is a lower bound for the sum since $g$ is convex and $\bar{u}_{2}<v_{0}$. When neither conditions hold, $u_{3} \geq u_{1}+u_{2}>\bar{u}_{1}+\bar{u}_{2}$, then by the convexity of $g$ and $\bar{u}_{1}+\bar{u}_{2}>v_{0}$, the summation is bounded from below by $\alpha_{3} g\left(\bar{u}_{1}+\bar{u}_{2}\right)$. 
Proposition 5.7. Consider the convex optimization problem (5.2) with $m=3, \ell=2, A=$ $(-1,1,1)$. Then

$$
\alpha_{1} f\left(u_{1}\right)+\alpha_{2} f\left(u_{2}\right)+\alpha_{3} g\left(u_{3}\right) \geq \min \left\{\alpha_{1} f\left(\bar{u}_{2}+\bar{u}_{3}\right), \alpha_{2} f\left(\bar{u}_{2}\right), \alpha_{3} g\left(\bar{u}_{3}\right)\right\},
$$

where

$$
\bar{u}_{2}=u_{0}-\frac{\alpha_{1} \alpha_{3} \alpha v_{0}}{\alpha_{1} \alpha_{2}+\alpha_{1} \alpha_{3}+\alpha_{2} \alpha_{3}}, \quad \bar{u}_{3}=\frac{\alpha_{3}\left(\alpha_{1}+\alpha_{2}\right) v_{0}}{\alpha_{1} \alpha_{2}+\alpha_{1} \alpha_{3}+\alpha_{2} \alpha_{3}} .
$$

Proof. Now the constraint is $u_{1} \geq u_{2}+u_{3}$. When $u_{2} \leq \bar{u}_{2}$, it follows from the convexity of $f$ and $\bar{u}_{2}<u_{0}$ that $\alpha_{2} f\left(\bar{u}_{2}\right)$ is a lower bound for the sum $\alpha_{1} f\left(u_{1}\right)+\alpha_{2} f\left(u_{2}\right)+\alpha_{3} g\left(u_{3}\right)$. Similarly, when $u_{3} \leq \bar{u}_{3}, \alpha_{3} g\left(\bar{u}_{3}\right)$ is a lower bound for the sum since $g$ is convex, $\bar{u}_{3}<v_{0}$. When neither conditions hold, $u_{1} \geq u_{2}+u_{3}>\bar{u}_{2}+\bar{u}_{3}>u_{0}$, then the summation is bounded from below by $\alpha_{1} f\left(\bar{u}_{2}+\bar{u}_{3}\right)$.

For convenience, define

$$
\begin{aligned}
G_{i j k}=\min & \left\{m_{i} m_{j} \mathfrak{E}\left(\frac{m_{j} \phi^{-\frac{2}{3}}+m_{i}(\pi-\phi)^{-\frac{2}{3}}}{m_{i}+m_{j}+m_{k}}, \phi\right), m_{j} m_{k} \mathfrak{E}\left(\frac{\left(m_{j}+m_{k}\right)(\pi-\phi)^{-\frac{2}{3}}}{m_{i}+m_{j}+m_{k}}, \pi-\phi\right),\right. \\
& \left.m_{i} m_{k} \mathfrak{E}\left((\pi-\phi)^{-\frac{2}{3}}+\frac{m_{j} \phi^{-\frac{2}{3}}}{m_{i}+m_{j}+m_{k}}, \pi-\phi\right)\right\}, \\
H_{i j k}=\min & \left\{m_{i} m_{k} \mathfrak{E}\left(\phi^{-\frac{2}{3}}+\frac{m_{j}(\pi-\phi)^{-\frac{2}{3}}}{m_{i}+m_{j}+m_{k}}, \phi\right), m_{i} m_{j} \mathfrak{E}\left(\phi^{-\frac{2}{3}}-\frac{m_{k}(\pi-\phi)^{-\frac{2}{3}}}{m_{i}+m_{j}+m_{k}}, \phi\right),\right. \\
J_{i j k}=\min & \left\{m_{j} m_{k} \mathfrak{E}\left(\frac{\left(m_{j}+m_{k}\right)(\pi-\phi)^{-\frac{2}{3}}}{m_{i}+m_{j}+m_{k}}, \pi-\phi\right)\right\}, \\
& m_{i} m_{k} \mathfrak{E}\left(\left(\frac{\left(m_{i}+m_{j}\right)(\pi-\phi)^{-\frac{2}{3}}}{m_{i}+m_{j}+m_{k}}, \pi-\phi\right), m_{j} m_{k} \mathfrak{E}\left(\frac{\left(m_{j}+m_{k}\right)(\pi-\phi)^{-\frac{2}{3}}}{m_{i}+m_{j}+m_{k}}, \pi-\phi\right),\right.
\end{aligned}
$$

Now we apply Propositions 5.6, 5.7 to estimate $\mathfrak{A}_{0}$ in (4.2) for the four-body problem.

Proposition 5.8. Given $\phi \in(0, \pi / 2]$ and $x \in P_{\phi, T, 4}$. Suppose

$$
x_{4}(0) \leq x_{3}(0) \leq x_{2}(0) \leq x_{1}(0) .
$$

Let $\mathfrak{E}$ be as in (2.11), $\left\{\mathbf{\Delta}_{0}^{\prime}, \mathbf{\Delta}_{1}^{\prime}, \mathbf{\Delta}_{2}^{\prime}\right\}$ be an ordered partition of subscript pairs. Given positive masses $m_{i}, i \in\{1,2,3,4\}$. Let $M$ be the total mass, and

$$
\mathcal{E}_{0}=\sum_{(i, j) \in \mathbf{\Lambda}_{1}^{\prime}} m_{i} m_{j} \mathfrak{E}\left(\left(M T^{2}\right)^{-\frac{1}{3}}\left|x_{i j}(0)\right|, \phi\right)+\sum_{(i, j) \in \mathbf{\Lambda}_{2}^{\prime}} m_{i} m_{j} \mathfrak{E}\left(\left(M T^{2}\right)^{-\frac{1}{3}}\left|x_{i j}(0)\right|, \pi-\phi\right) .
$$

Suppose $1 \leq i<j<k \leq 4$. Then $\mathcal{E}_{0}$ has lower bounds given as follows: 
(a) If $(i, j) \in \mathbf{\Delta}_{1}^{\prime},(j, k),(i, k) \in \mathbf{\Delta}_{2}^{\prime}$, then $\varepsilon_{0} \geq G_{i j k}$.

(b) If $(j, k) \in \mathbf{\Delta}_{1}^{\prime},(i, j),(i, k) \in \mathbf{\Delta}_{2}^{\prime}$, then $\varepsilon_{0} \geq G_{k j i}$.

(c) If $(i, j),(i, k) \in \mathbf{\Delta}_{1}^{\prime},(j, k) \in \mathbf{\Delta}_{2}^{\prime}$, then $\varepsilon_{0} \geq H_{i j k}$.

(d) If $(j, k),(i, k) \in \mathbf{\Delta}_{1}^{\prime},(i, j) \in \mathbf{\Delta}_{2}^{\prime}$, then $\varepsilon_{0} \geq H_{k j i}$.

(e) If $(i, j),(j, k),(i, k) \in \mathbf{\Delta}_{2}^{\prime}$, then $\varepsilon_{0} \geq J_{i j k}$.

Proof. Fix $\phi \in(0, \pi / 2]$. Let

$$
f(u)=\mathfrak{E}\left(\left(M T^{2}\right)^{-\frac{1}{3}} u, \phi\right), \quad g(u)=\mathfrak{E}\left(\left(M T^{2}\right)^{-\frac{1}{3}} u, \pi-\phi\right) .
$$

Then by Corollary 2.1,

$$
f(u)=\left(M^{2} T\right)^{-\frac{1}{3}} \mathfrak{E}_{M, T}(u, \phi), \quad g(u)=\left(M^{2} T\right)^{-\frac{1}{3}} \mathfrak{E}_{M, T}(u, \pi-\phi),
$$

and $f$ has global minimum value 0 at $u_{0}=\left(M T^{2}\right)^{1 / 3} \phi^{-2 / 3}, g$ has global minimum value 0 at $v_{0}=\left(M T^{2}\right)^{1 / 3}(\pi-\phi)^{-2 / 3}$, which is less than or equal to $u_{0}$. Note that $x_{i j}(0)+x_{j k}(0)=$ $x_{i k}(0)$.

The lower bound in (a) is obtained by Proposition 5.6(b) with

$$
\begin{aligned}
& \left(u_{1}, u_{2}, u_{3}\right)=\left(x_{i j}(0), x_{j k}(0), x_{i k}(0)\right), \\
& \left(\alpha_{1}, \alpha_{2}, \alpha_{3}\right)=\left(m_{i} m_{j}, m_{j} m_{k}, m_{i} m_{k}\right) .
\end{aligned}
$$

The lower bound in (b) is obtained by Proposition 5.6(b) with

$$
\begin{aligned}
& \left(u_{1}, u_{2}, u_{3}\right)=\left(x_{j k}(0), x_{i j}(0), x_{i k}(0)\right), \\
& \left(\alpha_{1}, \alpha_{2}, \alpha_{3}\right)=\left(m_{j} m_{k}, m_{i} m_{j}, m_{i} m_{k}\right) .
\end{aligned}
$$

The lower bound in (c) is obtained by Proposition 5.7 with

$$
\begin{aligned}
& \left(u_{1}, u_{2}, u_{3}\right)=\left(x_{i k}(0), x_{i j}(0), x_{j k}(0)\right), \\
& \left(\alpha_{1}, \alpha_{2}, \alpha_{3}\right)=\left(m_{i} m_{k}, m_{i} m_{j}, m_{j} m_{k}\right) .
\end{aligned}
$$

The lower bound in $(\mathrm{d})$ is obtained by Proposition 5.7 with

$$
\begin{aligned}
& \left(u_{1}, u_{2}, u_{3}\right)=\left(x_{i k}(0), x_{j k}(0), x_{i j}(0)\right), \\
& \left(\alpha_{1}, \alpha_{2}, \alpha_{3}\right)=\left(m_{i} m_{k}, m_{j} m_{k}, m_{i} m_{j}\right) .
\end{aligned}
$$

The lower bound in (e) is obtained by Proposition 5.1 with

$$
\begin{aligned}
& \left(u_{1}, u_{2}, u_{3}\right)=\left(x_{i j}(0), x_{j k}(0), x_{i k}(0)\right), \\
& \left(\alpha_{1}, \alpha_{2}, \alpha_{3}\right)=\left(m_{i} m_{j}, m_{j} m_{k}, m_{i} m_{k}\right) .
\end{aligned}
$$

Thus, we complete the proof. 
Following the proof for Proposition 5.8, one immediately obtains the following estimate for $\mathfrak{B}_{T}$ in (4.2) by replacing $x_{i}$ by $x_{\sigma_{i}}$.

Proposition 5.9. Given $\phi \in(0, \pi / 2], x \in P_{\phi, T, 4}$, and $\sigma \in S_{4}$. Suppose

$$
e^{-i \phi} x_{\sigma_{4}}(T) \leq e^{-i \phi} x_{\sigma_{3}}(T) \leq e^{-i \phi} x_{\sigma_{2}}(T) \leq e^{-i \phi} x_{\sigma_{1}}(T) .
$$

Let $\mathfrak{E}$ be as in (2.11), $\left\{\mathbf{\Lambda}_{0}^{\prime}, \mathbf{\Delta}_{1}^{\prime}, \mathbf{\Delta}_{2}^{\prime}\right\}$ be an ordered partition of subscript pairs. Given positive masses $m_{i}, i \in\{1,2,3,4\}$. Let $M$ be the total mass, and

$$
\mathcal{E}_{T}=\sum_{(i, j) \in \mathbf{\Lambda}_{1}^{\prime}} m_{i} m_{j} \mathfrak{E}\left(\left(M T^{2}\right)^{-\frac{1}{3}}\left|x_{i j}(T)\right|, \phi\right)+\sum_{(i, j) \in \mathbf{\Lambda}_{2}^{\prime}} m_{i} m_{j} \mathfrak{E}\left(\left(M T^{2}\right)^{-\frac{1}{3}}\left|x_{i j}(T)\right|, \pi-\phi\right) .
$$

Suppose $1 \leq i<j<k \leq 4$. Then $\mathcal{E}_{0}$ has lower bounds given as follows.

(a) If $\left(\sigma_{i}, \sigma_{j}\right) \in \mathbf{\Delta}_{1}^{\prime},\left(\sigma_{j}, \sigma_{k}\right),\left(\sigma_{i}, \sigma_{k}\right) \in \mathbf{\Delta}_{2}^{\prime}$, then $\mathcal{E}_{T} \geq G_{\sigma_{i} \sigma_{j} \sigma_{k}}$.

(b) If $\left(\sigma_{j}, \sigma_{k}\right) \in \mathbf{\Delta}_{1}^{\prime},\left(\sigma_{i}, \sigma_{j}\right),\left(\sigma_{i}, \sigma_{k}\right) \in \mathbf{\Delta}_{2}^{\prime}$, then $\mathcal{E}_{T} \geq G_{\sigma_{k} \sigma_{j} \sigma_{i}}$.

(c) If $\left(\sigma_{i}, \sigma_{j}\right),\left(\sigma_{i}, \sigma_{k}\right) \in \mathbf{\Delta}_{1}^{\prime},\left(\sigma_{j}, \sigma_{k}\right) \in \mathbf{\Delta}_{2}^{\prime}$, then $\mathcal{E}_{T} \geq H_{\sigma_{i} \sigma_{j} \sigma_{k}}$.

(d) If $\left(\sigma_{j}, \sigma_{k}\right),\left(\sigma_{i}, \sigma_{k}\right) \in \mathbf{\Delta}_{1}^{\prime},\left(\sigma_{i}, \sigma_{j}\right) \in \mathbf{\Delta}_{2}^{\prime}$, then $\mathcal{E}_{T} \geq H_{\sigma_{k} \sigma_{j} \sigma_{i}}$.

(e) If $\left(\sigma_{i}, \sigma_{j}\right),\left(\sigma_{j}, \sigma_{k}\right),\left(\sigma_{i}, \sigma_{k}\right) \in \mathbf{\Delta}_{2}^{\prime}$, then $\varepsilon_{0} \geq J_{\sigma_{i} \sigma_{j} \sigma_{k}}$.

\section{Application to the four-body problem}

Although we only focus on the four-body problem here, our estimates, which are based on Theorem 4.1 and properties of the Keplerian action functional, can be easily applied to general $n$-body problems. We wish that the application shown in this section will motivate many further applications.

As mentioned in Section 4, given positive masses $\left(m_{1}, \cdots, m_{n}\right)$, turning angle $\phi \in$ $(0, \pi / 2]$, transfer time $T>0$, and $\sigma \in S_{n}$, there exist classical solutions for (1.1) in a component $\mathbf{b}^{\sigma}$ of $P_{\phi, T, n}^{\sigma}$ as soon as we prove

$$
\inf _{\mathbf{b}^{\sigma}} \mathcal{A}_{T}<\inf _{\partial P_{\phi, T, n}^{\sigma}} \mathcal{A}_{T}
$$

If we are able to find a suitable collision-free test path in $\mathbf{b}^{\sigma}$ that has even lower action value, then the inequality holds for an open set of masses and turning angles.

For the four-body problem, there are 6 pairs of $(i, j)$ with $i<j$ and $2^{6}$ combinations of $\boldsymbol{\Delta}_{0}$. If we classify collision paths according to the set $\boldsymbol{\Delta}_{0}$ of collision pairs, then there is a total of 63 cases, so an obtuse and thoughtless lower bound estimate for the right-hand side of the above inequality could be obtained by providing estimate for each individual case and then taking their minimum value. This manifests the merit of using ordered 
partitions in place of standard ordered partition for subscript pairs in Theorem 4.1. By choosing ordered partition for subscript pairs, it is sufficient to check the 6 cases with single colliding pair. This will be illustrated in the Theorem 6.1.

In order to shorten our expressions, we fix positive masses $\left(m_{1}, m_{2}, m_{3}, m_{4}\right)$, the turning angle $\phi$, and use notations $E_{i j k \ell}, F_{i j k \ell}, G_{i j k}, H_{i j k}$ defined in (5.3), (5.4). Given $\sigma \in S_{4}$, $1 \leq k<\ell \leq 4$. Define

$$
\begin{aligned}
& C_{k \ell}^{\sigma} \\
& = \begin{cases}m_{k} m_{\ell}\left(\pi^{\frac{2}{3}}-\phi^{\frac{2}{3}}\right)+\sum_{\substack{i<j \\
\sigma_{i}^{-1}<\sigma_{j}^{-1}}} m_{i} m_{j} \phi^{\frac{2}{3}}+\sum_{\substack{i<j \\
\sigma_{i}^{-1}>\sigma_{j}^{-1}}} m_{i} m_{j}(\pi-\phi)^{\frac{2}{3},} & \text { if } \sigma_{k}^{-1}<\sigma_{\ell}^{-1}, \\
m_{k} m_{\ell}\left(\pi^{\frac{2}{3}}-(\pi-\phi)^{\frac{2}{3}}\right)+\sum_{\substack{i<j \\
\sigma_{i}^{-1}<\sigma_{j}^{-1}}} m_{i} m_{j} \phi^{\frac{2}{3}}+\sum_{\substack{i<j \\
\sigma_{i}^{-1}>\sigma_{j}^{-1}}} m_{i} m_{j}(\pi-\phi)^{\frac{2}{3}}, & \text { if } \sigma_{k}^{-1}>\sigma_{\ell}^{-1},\end{cases} \\
& D_{k \ell}=m_{k} m_{\ell}\left(\pi^{\frac{2}{3}}-\phi^{\frac{2}{3}}\right)+\sum_{i<j} m_{i} m_{j} \phi^{\frac{2}{3}} .
\end{aligned}
$$

If $x \in \partial P_{\phi, T, 4}^{\sigma}$ and the ordered partition $\left\{\mathbf{\Delta}_{0}^{\prime}, \mathbf{\Delta}_{1}^{\prime}, \mathbf{\Delta}_{2}^{\prime}\right\}$ is as (4.3), then $\frac{3}{2} C_{12}^{\sigma}$ is exactly the first line of $\mathfrak{A}_{\tau}$ defined in Theorem 4.1.

Here is our application to the four-body problem:

Theorem 6.1. Given $T>0$. Consider $\sigma=(1243)$ and the triple retrograde braid $\mathbf{b}^{\sigma}$ as shown in Fig. 4. There exist an open set $\mathcal{M}$ of positive masses $\left(m_{1}, m_{2}, m_{3}, m_{4}\right)$ containing $(1,1,1,1)$ and an open set $\Phi$ of turning angles $\phi \in(0, \pi / 2]$ containing $\pi / 2$ such that there exist classical solutions for the four-body problem which minimizes the action functional on the component $\mathbf{b}^{\sigma}$ of $\mathbf{b}^{\sigma} \subset P_{\phi, T, 4}$.

In particular, if $C_{k \ell}^{\sigma}, D_{k \ell}, E_{i j k \ell}, F_{i j k \ell}, G_{i j k}, H_{i j k}$ are as in (6.1a), (5.3), (5.4), then such solutions exist provided

$$
\inf _{\partial P_{\phi, T, 4}^{\sigma}} \mathcal{A}_{T} \geq\left(\frac{T}{M}\right)^{\frac{1}{3}} \min \left\{\max \left\{A_{i j}, B_{i j}\right\}: 1 \leq i<j \leq 4\right\}
$$

where

$$
\begin{aligned}
& A_{12}=\frac{3}{2} C_{12}^{\sigma}+G_{134}, \\
& A_{34}=\frac{3}{2} C_{34}^{\sigma}+G_{421}, \\
& A_{23}=\frac{3}{2} C_{23}^{\sigma}+G_{134}, \\
& A_{13}=\frac{3}{2} C_{13}^{\sigma}+H_{243} \text {, } \\
& A_{24}=\frac{3}{2} C_{24}^{\sigma}+H_{213} \text {, } \\
& A_{14}=\frac{3}{2} C_{14}^{\sigma}+H_{243} \text {, } \\
& B_{12}=\frac{3}{2} D_{12}+E_{1234} \\
& B_{34}=\frac{3}{2} D_{34}+E_{4321} \text {, } \\
& B_{23}=\frac{3}{2} D_{23}+F_{2314} \text {, } \\
& B_{13}=\frac{3}{2} D_{13}+E_{1342} \\
& B_{24}=\frac{3}{2} D_{24}+E_{2413} \text {, } \\
& B_{14}=\frac{3}{2} D_{14}+F_{1423} \text {. }
\end{aligned}
$$

Proof. Given $x \in \overline{P_{\phi, T, 4}^{\sigma}}$, the weak closure of $P_{\phi, T, 4}^{\sigma}$, we have

$$
\{(1,3),(2,3),(2,4)\} \subset \mathbf{\Delta}_{1} \cup \mathbf{\Delta}_{0,1}, \quad\{(1,2),(1,4),(3,4)\} \subset \boldsymbol{\Delta}_{2} \cup \mathbf{\Delta}_{0,2} .
$$


Note that a subscript pair $(k, \ell), k<\ell$, belongs to the set $\boldsymbol{\Delta}_{1} \cup \boldsymbol{\Delta}_{0,1}$ of order-preserving pairs if and only if $\sigma_{k}^{-1}<\sigma_{\ell}^{-1}$. The constraint by $\sigma$ implies that

$$
e^{-i \phi} x_{3}(T) \leq e^{-i \phi} x_{1}(T) \leq e^{-i \phi} x_{4}(T) \leq e^{-i \phi} x_{2}(T) .
$$

If $x \in \partial P_{\phi, T, 4}^{\sigma}$, then $x$ is a collision path; i.e. $\mathbf{\Delta}_{0} \neq \varnothing$.

The table below lists the six possibilities and our selected ordered partitions for subscript pairs.

\begin{tabular}{||c|c|c|c||}
\hline $\mathbf{\Delta}_{0}$ & $\mathbf{\Delta}_{0}^{\prime}$ & $\mathbf{\Delta}_{1}^{\prime}$ & $\mathbf{\Delta}_{2}^{\prime}$ \\
\hline$(1,2) \in \mathbf{\Delta}_{0}$ & $\{(1,2)\}$ & $\{(1,3),(2,3),(2,4)\}$ & $\{(1,4),(3,4)\}$ \\
\hline$(3,4) \in \mathbf{\Delta}_{0}$ & $\{(3,4)\}$ & $\{(1,3),(2,3),(2,4)\}$ & $\{(1,2),(1,4)\}$ \\
\hline$(2,3) \in \mathbf{\Delta}_{0}$ & $\{(2,3)\}$ & $\{(1,3),(2,4)\}$ & $\{(1,2),(1,4),(3,4)\}$ \\
\hline$(1,3) \in \mathbf{\Delta}_{0}$ & $\{(1,3)\}$ & $\{(2,3),(2,4)\}$ & $\{(1,2),(1,4),(3,4)\}$ \\
& $=\left\{\left(\sigma_{3}, \sigma_{4}\right)\right\}$ & $=\left\{\left(\sigma_{1}, \sigma_{4}\right),\left(\sigma_{1}, \sigma_{2}\right)\right\}$ & $=\left\{\left(\sigma_{1}, \sigma_{3}\right),\left(\sigma_{2}, \sigma_{3}\right),\left(\sigma_{2}, \sigma_{4}\right)\right\}$ \\
\hline$(2,4) \in \mathbf{\Delta}_{0}$ & $\{(2,4)\}$ & $\{(1,3),(2,3)\}$ & $\{(1,2),(1,4),(3,4)\}$ \\
& $=\left\{\left(\sigma_{1}, \sigma_{2}\right)\right\}$ & $=\left\{\left(\sigma_{3}, \sigma_{4}\right),\left(\sigma_{1}, \sigma_{4}\right)\right\}$ & $=\left\{\left(\sigma_{1}, \sigma_{3}\right),\left(\sigma_{2}, \sigma_{3}\right),\left(\sigma_{2}, \sigma_{4}\right)\right\}$ \\
\hline$(1,4) \in \mathbf{\Delta}_{0}$ & $\{(1,4)\}$ & $\{(1,3),(2,3),(2,4)\}$ & $\{(1,2),(3,4)\}$ \\
& $=\left\{\left(\sigma_{2}, \sigma_{3}\right)\right\}$ & $=\left\{\left(\sigma_{3}, \sigma_{4}\right),\left(\sigma_{1}, \sigma_{4}\right),\left(\sigma_{1}, \sigma_{2}\right)\right\}$ & $=\left\{\left(\sigma_{1}, \sigma_{3}\right),\left(\sigma_{2}, \sigma_{4}\right)\right\}$ \\
\hline
\end{tabular}

To estimate $\mathfrak{A}_{0}$ or $\mathfrak{A}_{T}$ in Theorem 4.1, we apply Proposition 5.8 for the first 3 cases, apply Proposition 5.9 for the last 3 cases. To estimate $\mathfrak{B}_{0}$ or $\mathfrak{B}_{T}$ in Theorem 4.1 , we apply Proposition 5.3 for the first 3 cases, apply Proposition 5.4 for the last 3 cases.

In the first case, $(1,2) \in \boldsymbol{\Lambda}_{0}, \mathfrak{A}_{0}$ is bounded from below by $A_{12}$ and $\mathfrak{B}_{0}$ is bounded from below by $B_{12}$, where

$$
\begin{aligned}
& A_{12}=\frac{3}{2}\left[m_{1} m_{2} \pi^{\frac{2}{3}}+\left(m_{1} m_{3}+m_{2} m_{3}+m_{2} m_{4}\right) \phi^{\frac{2}{3}}+\left(m_{1} m_{4}+m_{3} m_{4}\right)(\pi-\phi)^{\frac{2}{3}}\right]+G_{134} \\
& B_{12}=\frac{3}{2}\left[m_{1} m_{2} \pi^{\frac{2}{3}}+\left(m_{1} m_{3}+m_{2} m_{3}+m_{2} m_{4}+m_{1} m_{4}+m_{3} m_{4}\right) \phi^{\frac{2}{3}}\right]+E_{1234} .
\end{aligned}
$$

Then $\max \left\{A_{12}, B_{12}\right\}$ is a lower bound for $(M / T)^{\frac{1}{3}} \mathcal{A}_{T}(x)$ in this case.

Other cases are similar: $\max \left\{A_{i j}, B_{i j}\right\}$ is a lower bound for $(M / T)^{\frac{1}{3}} \mathcal{A}_{T}(x)$ in the case $(i, j) \in \mathbf{\Delta}_{0}$, where

$$
\begin{aligned}
& A_{34}=\frac{3}{2}\left[m_{3} m_{4} \pi^{\frac{2}{3}}+\left(m_{1} m_{3}+m_{2} m_{3}+m_{2} m_{4}\right) \phi^{\frac{2}{3}}+\left(m_{1} m_{2}+m_{1} m_{4}\right)(\pi-\phi)^{\frac{2}{3}}\right]+G_{421}, \\
& B_{34}=\frac{3}{2}\left[m_{3} m_{4} \pi^{\frac{2}{3}}+\left(m_{1} m_{3}+m_{2} m_{3}+m_{2} m_{4}+m_{1} m_{2}+m_{1} m_{4}\right) \phi^{\frac{2}{3}}\right]+E_{4321}, \\
& A_{23}=\frac{3}{2}\left[m_{2} m_{3} \pi^{\frac{2}{3}}+\left(m_{1} m_{3}+m_{2} m_{4}\right) \phi^{\frac{2}{3}}+\left(m_{1} m_{2}+m_{1} m_{4}+m_{3} m_{4}\right)(\pi-\phi)^{\frac{2}{3}}\right]+G_{134}, \\
& B_{23}=\frac{3}{2}\left[m_{2} m_{3} \pi^{\frac{2}{3}}+\left(m_{1} m_{3}+m_{2} m_{4}+m_{1} m_{2}+m_{1} m_{4}+m_{3} m_{4}\right) \phi^{\frac{2}{3}}\right]+F_{2314}, \\
& A_{13}=\frac{3}{2}\left[m_{1} m_{3} \pi^{\frac{2}{3}}+\left(m_{2} m_{3}+m_{2} m_{4}\right) \phi^{\frac{2}{3}}+\left(m_{1} m_{2}+m_{1} m_{4}+m_{3} m_{4}\right)(\pi-\phi)^{\frac{2}{3}}\right]+H_{243},
\end{aligned}
$$




$$
\begin{aligned}
& B_{13}=\frac{3}{2}\left[m_{1} m_{3} \pi^{\frac{2}{3}}+\left(m_{2} m_{3}+m_{2} m_{4}+m_{1} m_{2}+m_{1} m_{4}+m_{3} m_{4}\right) \phi^{\frac{2}{3}}\right]+E_{1342}, \\
& A_{24}=\frac{3}{2}\left[m_{2} m_{4} \pi^{\frac{2}{3}}+\left(m_{1} m_{3}+m_{2} m_{3}\right) \phi^{\frac{2}{3}}+\left(m_{1} m_{2}+m_{1} m_{4}+m_{3} m_{4}\right)(\pi-\phi)^{\frac{2}{3}}\right]+H_{213} \\
& B_{24}=\frac{3}{2}\left[m_{2} m_{4} \pi^{\frac{2}{3}}+\left(m_{1} m_{3}+m_{2} m_{3}+m_{1} m_{2}+m_{1} m_{4}+m_{3} m_{4}\right) \phi^{\frac{2}{3}}\right]+E_{2413} \\
& A_{14}=\frac{3}{2}\left[m_{1} m_{4} \pi^{\frac{2}{3}}+\left(m_{1} m_{3}+m_{2} m_{3}+m_{2} m_{4}\right) \phi^{\frac{2}{3}}+\left(m_{1} m_{2}+m_{3} m_{4}\right)(\pi-\phi)^{\frac{2}{3}}\right]+H_{243}, \\
& B_{14}=\frac{3}{2}\left[m_{1} m_{4} \pi^{\frac{2}{3}}+\left(m_{1} m_{3}+m_{2} m_{3}+m_{2} m_{4}+m_{1} m_{2}+m_{3} m_{4}\right) \phi^{\frac{2}{3}}\right]+F_{1423} .
\end{aligned}
$$

In terms of $C_{k, \ell}^{\sigma}, D_{k \ell}$ defined in (6.1a), these are exactly the $A_{i j}{ }^{\prime}$ s, $B_{i j}$ 's defined in the statement of the proposition. At least one of these six cases occur, so the minimum of the six $\max \left\{A_{i j}, B_{i j}\right\}$ is clearly a lower bound for $(M / T)^{\frac{1}{3}} \mathcal{A}_{T}(x)$.

Now, to complete the proof of the theorem, by continuity it is sufficient to prove that the inequality (6.2) holds for the special choice $\left(m_{1}, m_{2}, m_{3}, m_{4}, \phi\right)=(1,1,1,1, \pi / 2)$ of masses and turning angle. We may just pick a suitable test path in $\mathbf{b}^{\sigma}$ for this case and calculate its action value. For this case we have the following formula for $\mathfrak{E}(\rho, \phi)$ :

$$
\begin{aligned}
\mathfrak{E}\left(\rho, \frac{\pi}{2}\right) & =\Im_{1,1}\left(\rho, \frac{\pi}{2}\right)-\frac{3}{2}\left(\frac{\pi}{2}\right)^{\frac{2}{3}} \\
& =\frac{1}{2} \inf _{\Gamma_{2}(0,2 \rho)} I_{1,2}-\frac{3}{2}\left(\frac{\pi}{2}\right)^{\frac{2}{3}} .
\end{aligned}
$$

The first line is simply the definition of $\mathfrak{E}$ in (2.11). The second line holds because, for fixed $\rho$, the minimizing Keplerian arc for $\Im_{1,1}(\rho, \pi / 2)$ connects one end of the latus rectum to the pericentre, and by reflecting the arc with respect to the major axis we obtain a Keplerian arc connecting two ends of the latus rectum, which has length $2 \rho$. Since the action integral is a Lambert parameter (Proposition 2.2), the action value of this extended Keplerian arc is exactly the same as the rectilinear Keplerian arc ejecting from the origin and reaches $2 \rho$ at time 2, as they already have three Lambert parameters in common (using notations in Subsection 2.2, they are $\left|\xi_{0}\right|+\left|\xi_{1}\right|,\left|\xi_{0}-\xi_{1}\right|$, and transfer time). The action value of this extended Keplerian arc is therefore $2 \mathfrak{I}_{1,1}(\rho, \pi / 2)=\inf _{\Gamma_{2}(0,2 \rho)} I_{1,2}$, and the original minimizing Keplerian arc has half of this action.

Bearing this in mind, for our particular choice of masses and turning angle, we find the values of $A_{i j}$ 's and $B_{i j}$ 's are:

$$
\begin{aligned}
& A_{12}=A_{34}=A_{23}=A_{13}=A_{24}=A_{14} \approx 13.4047, \\
& B_{12}=B_{34}=B_{13}=B_{24} \approx 13.4122, \\
& B_{23}=B_{14} \approx 13.4201 .
\end{aligned}
$$

With the help of formulae (2.7a), (2.7b), (2.8), these quantities can be obtained at any desired precision. Now $\min \left\{\max \left\{A_{i j}, B_{i j}\right\}: 1 \leq i<j \leq 4\right\} \approx 13.4122$, accurate to the fourth decimal place. 
Fix $T=1 / 2$. The test path selected is $x_{\text {test }}=\left(x_{1}, x_{2}, x_{3}, x_{4}\right)$, where:

$$
\begin{aligned}
& x_{1}(t)=(0.6464 \cos (\pi t)-0.0117 \cos (3 \pi t),-0.1824 \sin (\pi t)-0.0035 \sin (3 \pi t)), \\
& x_{2}(t)=(0.1824 \cos (\pi t)-0.0035 \cos (3 \pi t), 0.6464 \sin (\pi t)+0.0117 \sin (3 \pi t)), \\
& x_{3}(t)=-x_{2}(t), \\
& x_{4}(t)=-x_{1}(t) .
\end{aligned}
$$

Its action value, accurate to the fourth decimal place, is 6.7001, which is less than

$$
\left(\frac{T}{M}\right)^{\frac{1}{3}} \min \left\{\max \left\{A_{i j}, B_{i j}\right\}: 1 \leq i<j \leq 4\right\} \approx 6.7061 .
$$

This completes our proof.

Remark 6.1. We remark that the open sets $\mathcal{M}$ and $\Phi$ depends on the choice of braids, and the values of $A_{i j}$ 's and $B_{i j}$ 's are independent of the transfer time $T$.

It is often possible to find multiple ways of choosing lower bounds $A_{i j}, B_{i j}$ for $\mathfrak{A}_{\tau}, \mathfrak{B}_{\tau}$ by using Propositions 5.3, 5.4, 5.8, and 5.9. For example, in Theorem 6.1 we may choose

$$
A_{23}=\frac{3}{2} C_{23}^{\sigma}+G_{421}, \quad A_{14}=\frac{3}{2} C_{14}^{\sigma}+H_{421} .
$$

One may find all applicable combinations and choose the largest one. In fact, such lower bound estimates can be further improved using Proposition 5.5. In order to make it simple and clear, we do not pursuit for optimal bounds here.

Remark 6.2. In the proof we have used the fact that the action integral is a Lambert parameter to deduce

$$
\mathfrak{I}_{1,1}\left(\rho, \frac{\pi}{2}\right)=\frac{1}{2} \inf _{\Gamma_{2}(0,2 \rho)} I_{1,2}
$$

Following the same argument, one can easily generalize it to more general $\phi \in\left(0, \frac{\pi}{2}\right]$ :

$$
\mathfrak{I}_{1,1}(\rho, \phi)=\frac{1}{2} \inf _{\Gamma_{2}\left(x_{0}, x_{1}\right)} I_{1,2}=\frac{1}{2}\left(\inf _{\Gamma_{2}\left(0, x_{1}\right)} I_{1,2}-\inf _{\Gamma_{2}\left(0, x_{0}\right)} I_{1,2}\right),
$$

where $x_{0}=\rho(1-\sin \phi), x_{1}=\rho(1+\sin \phi)$. The last equation can be calculated easily by using (2.8).

Remark 6.3. Collecting numerical data, including the action value $\mathcal{A}_{\text {test }}$ of our test path, lower bound $\mathcal{A}_{\text {coll }}$ for $\inf _{\partial P_{\phi, T, n}^{\sigma}} \mathcal{A}_{T}$ obtained from Section 6, numerical value of $\mathcal{A}_{\text {inf }}=$ $\inf _{P_{\phi, T}^{\sigma}, n} \mathcal{A}_{T}$, and initial data of the action minimizer. In the proof we set $T=1 / 2$ so that the action minimizer has relative period 1. 


\begin{tabular}{||l|l|l|l|l||}
\hline$\left(m_{1}, m_{2}, m_{3}, m_{4}, \phi\right)$ & $\mathcal{A}_{\text {inf }}$ & $\mathcal{A}_{\text {test }}$ & $\mathcal{A}_{\text {coll }}$ & initial data $\left(\begin{array}{l}x_{1}, x_{2}, x_{3}, x_{4} \\
v_{1}, v_{2}, v_{3}, v_{4}\end{array}\right)$ \\
\hline$(1,1,1,1, \pi / 2)$ & 6.68209 & 6.70013 & 6.70610 & $\left(\begin{array}{c}0.639533,0.181359,-0.181359,-0.639533 \\
-0.661797 i, 2.333714 i,-2.333714 i, 0.661797 i\end{array}\right)$ \\
\hline
\end{tabular}

\section{Acknowledgements}

It is my great honor and pleasure to contribute in this special volume dedicated to Professor Paul Rabinowitz on his 80th birthday. Paul's research work were great inspiration for my research interests, and his generous support and recognition have greatly influenced my research career. I also thank Jaeyoung Byeon, Yiming Long, and Zhi-Qiang Wang for their kind invitation to the Jeju meeting and this special issue. This work is supported in parts by the Ministry of Science and Technology in Taiwan.

\section{References}

[1] A. Albouy, Lectures on the two-body problem, Classical and Celestial Mechanics, (Recife, 1993/1999), 63-116, Princeton University Press, Princeton, NJ, 2002.

[2] G. Arioli, F. Gazzola and S. Terracini, Minimization properties of Hill's orbits and applications to some $N$-body problems, Ann. Inst. H. Poincaré Anal. Non Linéaire, 17 (2000), 617-650.

[3] G. Arioli, V. Barutello and S. Terracini, A new branch of Mountain Pass solutions for the choreographical 3-body problem, Commun. Math. Phys., 268 (2006), 439-463.

[4] V. Barutello, D. Ferrario and S. Terracini, Symmetry groups of the planar three-body problem and action-minimizing trajectories, Arch. Ration. Mech. Anal.,, 190 (2008), 189-226.

[5] K.-C. Chen, Action-minimizing orbits in the parallelogram four-body problem with equal masses, Arch. Ration. Mech. Anal., 158 (2001), 293-318.

[6] K.-C. Chen, Binary decompositions for the planar $N$-body problem and symmetric periodic solutions, Arch. Ration. Mech. Anal., 170 (2003), 247-276.

[7] K.-C. Chen, Variational methods on periodic and quasi-periodic solutions for the $N$-body problem, Ergodic Theory Dynam. Syst., 23 (2003), 1691-1715.

[8] K.-C. Chen, Removing collision singularities from action minimizers for the $\mathrm{N}$-body problem with free boundaries, Arch. Ration. Mech. Anal., 181 (2006), 311-331.

[9] K.-C. Chen, Existence and minimizing properties of retrograde orbits to the three-body problem with various choices of masses, Ann. Math., 167 (2008), 325-348.

[10] K.-C. Chen, and Y.-C. Lin, On action-minimizing retrograde and prograde orbits of the threebody problem, Commun. Math. Phys., 291 (2009), 403-441.

[11] K.-C. Chen, T. Ouyang, and Z. Xia, Action-minimizing periodic and quasi-periodic solutions in the $n$-body problem, Math. Res. Lett., 19 (2012), 483-497.

[12] A. Chenciner, Action minimizing solutions in the Newtonian $n$-body problem: from homology to symmetry, Proceedings of the International Congress of Mathematicians, (Beijing, 2002), Vol III, 279-294. Errata, Proceedings of the International Congress of Mathematicians, (Beijing, 2002). Vol I, 651-653.

[13] A. Chenciner, J. Gerver, R. Montgomery, and C. Simó, Simple choreographic motions of N bodies: a preliminary study, Geometry, Mechanics, and Dynamics, 287-308, Springer, New York, 2002. 
[14] A. Chenciner, and J. Fejoz, Unchained polygons and the N-body problem, Regul. Chaotic Dyn., 14 (2009), 64-115.

[15] A. Chenciner and R. Montgomery, A remarkable periodic solution of the three-body problem in the case of equal masses, Ann. Math., 152 (2000), 881-901.

[16] A. Chenciner, and A. Venturelli, Minima de l'intégrale d'action du Problème newtonien de 4 corps de masses égales dans $\mathbb{R}^{3}$ : orbites "hip-hop". Celestial Mech. Dynam. Astronomy, 77(2) (2000), 139-152.

[17] D. Ferrario, Transitive decomposition of symmetry groups for the $n$-body problem, Adv. Math., 213 (2007), 763-784.

[18] D. Ferrario, and S. Terracini, On the existence of collisionless equivariant minimizers for the classical $n$-body problem, Invent. Math., 155 (2004), 305-362.

[19] G. Fusco, G. F. Gronchi, and P. Negrini, Platonic polyhedra, topological constraints and periodic solutions of the classical N-body problem, Invent. Math., 185 (2011), 283-332.

[20] W. Gordon, A minimizing property of Keplerian orbits, Amer. J. Math., 99 (1977), 961-971.

[21] T. Kapela, and C. Simó, Computer assisted proofs for nonsymmetric planar choreographies and for stability of the Eight, Nonlinearity, 20 (2007), 1241-1255.

[22] T. Kapela, and P. Zgliczyński, The existence of simple choreographies for the $N$-body problem-a computer-assisted proof, Nonlinearity, 16 (2003), 1899-1918.

[23] J. H. Lambert, Insigniores orbitae cometarum proprietates, Augustae Vindelicorum (Augsburg), (1761).

[24] J. L. Lagrange, Sur une manière particulière d'exprimer le temps dans les sections coniques, décrites par des forces tendantes au foyer et réciproquement proportionnelles aux carrés des distances, CEuvres, 4 (1778), 559-582.

[25] C. Marchal, The family $P_{12}$ of the three-body problem-the simplest family of periodic orbits, with twelve symmetries per period, Celestial Mech. Dynam. Astronom., 78 (2000), 279-298.

[26] R. Montgomery, The $N$-body problem, the braid group, and action-minimizing periodic solutions, Nonlinearity, 11 (1998), 363-376.

[27] C. Moore, Braids in classical dynamics, Phy. Rev. Lett., 70 (1993), 3675-3679.

[28] C. Simó, New families of solutions in N-body problems, European Congress of Mathematics, Vol. I (Barcelona, 2000), 101-115, Progr. Math., 201, Birkhauser, Basel, 2001.

[29] C. Simó, Choreographic solutions of the planar three body problem, http://www.maia.ub.es/dsg/3body.html.

[30] S. Terracini and A. Venturelli, Symmetric trajectories for the $2 N$-body problem with equal masses, Arch. Rat. Mech. Anal., 184 (2007), 465-493.

[31] A. Venturelli, Application de la minimisation de l'action au problème des $N$ corps dans le plan et dans l'espace, Thesis, Université de Paris 7, 2002. 